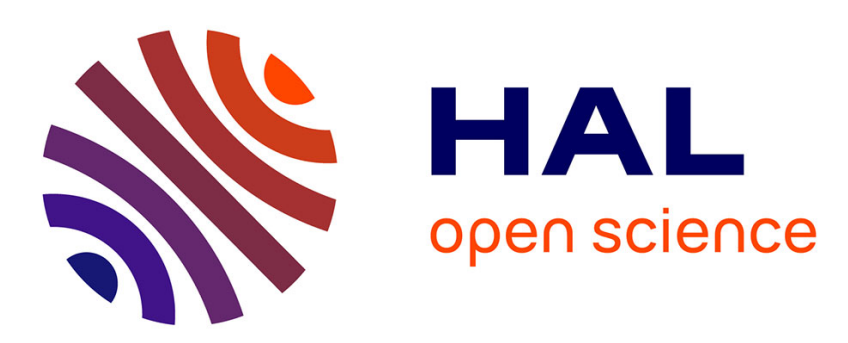

\title{
Stochastic modeling of chemical-mechanical coupling in striated muscles
}

Matthieu Caruel, Philippe Moireau, Dominique Chapelle

\section{To cite this version:}

Matthieu Caruel, Philippe Moireau, Dominique Chapelle. Stochastic modeling of chemical-mechanical coupling in striated muscles. Biomechanics and Modeling in Mechanobiology, 2019, 10.1007/s10237018-1102-z . hal-01928279v2

\section{HAL Id: hal-01928279 \\ https://hal.inria.fr/hal-01928279v2}

Submitted on 21 Dec 2018

HAL is a multi-disciplinary open access archive for the deposit and dissemination of scientific research documents, whether they are published or not. The documents may come from teaching and research institutions in France or abroad, or from public or private research centers.
L'archive ouverte pluridisciplinaire HAL, est destinée au dépôt et à la diffusion de documents scientifiques de niveau recherche, publiés ou non, émanant des établissements d'enseignement et de recherche français ou étrangers, des laboratoires publics ou privés. 


\title{
Stochastic modeling of chemical-mechanical coupling in striated muscles
}

\author{
Matthieu Caruel, Philippe Moireau, Dominique Chapelle
}

Received: 15 May 2018 / Accepted: 21 November 2018

\begin{abstract}
We propose a chemical-mechanical model of myosin heads in sarcomeres, within the classical description of rigid sliding filaments. In our case, myosin heads have two mechanical degrees-of-freedom (dofs) one of which associated with the so-called power stroke - and two possible chemical states, i.e. bound to an actin site or not. Our major motivations are twofold: (1) to derive a multiscale coupled chemical-mechanical model, and (2) to thus account - at the macroscopic scale - for mechanical phenomena that are out of reach for classical muscle models. This model is first written in the form of Langevin stochastic equations, and we are then able to obtain the corresponding Fokker-Planck partial differential equations governing the probability density functions associated with the mechanical dofs and chemical states. This second form is important, as it allows to monitor muscle energetics, and also to compare our model with classical ones, such as the Huxley'57 model to which our equations are shown to reduce under two different types of simplifying assumptions. This provides insight, and gives a Langevin form for Huxley'57. We then show how we can calibrate our model based on experimental data - taken here for skeletal muscles - and numerical simulations demonstrate the adequacy of the model to represent complex physiological phenomena, in particular the fast isomet-
\end{abstract}

\section{Caruel}

MSME Laboratory, Université Paris-Est Créteil, CNRS

5 boulevard Descartes - Champs-sur-Marne - 77454 Marne-

la-Vallée Cedex 2, France

E-mail: matthieu.caruel@u-pec.fr

P. Moireau, D. Chapelle

Inria - Ecole Polytechnique, CNRS - Université Paris-Saclay

Inria Saclay-Ile-de-France - 1 Rue d'Estienne d'Orves, 91120

Palaiseau, France

E-mail: philippe.moireau@inria.fr

E-mail: dominique.chapelle@inria.fr ric transients in which the power stroke is known to have a crucial role, thus circumventing a limitation of many classical models.

Keywords: muscle modeling; sarcomere; sliding filament; cross-bridge; power stroke; Langevin equations; Fokker-Planck equations

\section{Introduction}

Muscle contraction occurs at the nanoscale of a hierarchical multi-scale structure with the attachment of socalled cross-bridges within sarcomeres, namely, the creation of chemical bonds between myosin heads and specific sites on actin filaments, under the influence of other chemical species, namely, calcium ions and ATP, in particular (Alberts et al 2014). Furthermore, to allow for possibly large and fast muscle length variations concurrent with active tension, the actin and myosin filaments slide along each other, while the myosin heads unbind and rebind at different actin sites (Huxley 1957). At a finer level of detail, it has been established that this binding-unbinding cycle has in fact four main stages, with two distinct stages associated with different angular configurations of the myosin head in each of the bound-unbound states (Lymn and Taylor 1971). The transition between the two angular positions in the bound state is called the power stroke, as it is accompanied by a surge in the active force developed in the cross-bridge.

In order to carry over this nanoscale behavior to the macroscale for muscle modeling purposes, an adequate multiscale approach must be undertaken. A classical strategy consists in using a statistical framework to describe the population of myosin heads, with probability densities associated with variables that repre- 
sent the state of individual myosins. These probabilities are then used to average microscopic quantities, hence to obtain the desired macroscopic physical quantities such as forces, energies, etc.. In so doing, various simplifying modeling assumptions can be introduced to obtain more tractable descriptions of the probability densities and/or direct expressions governing the macroscopic quantities. A very common assumption consists in considering the actin and myosin filaments as rigid, with sliding allowed by the deformation of myosin heads themselves - in addition to binding-unbinding - and with a periodic spacing of available actin sites along the actin filaments (Huxley 1957). Additional assumptions made in the pioneering Huxley'57 model consist in modeling an active cross-bridge as a linear spring, with chemical rates of binding and unbinding only dependent on the distance to the nearest actin site. The relevant remaining probability is then that for a myosin located at a given distance $s$ to the nearest actin site to be actually attached, in which case the induced force is in an affine relation with $s$. Note that this description does not include a concept of power stroke per se, since the deformation of a bound myosin head varies linearly during filament sliding. Nevertheless, this muscle model has proven to be quite amenable to numerical simulations, in particular when considering the moments of the probability - namely, integrals of this probability weighed by powers of $s$ - the first two moments being directly related to the overall active stiffness and stress induced at the macroscopic level by the cross-bridges. Closed-form evolution equations for these moments can be obtained by resorting to adequate closure relations (Zahalak 1981), or with specific choices of binding and unbinding rates (Chen and Hill 1974; Hill and Chen 1974; Bestel et al 2001; Guérin et al 2011). Furthermore, extensions have been proposed to incorporate this type of model into a general 3D continuum mechanics formalism compatible with large strains and complex anisotropic constitutive behaviors, with the motivation of enabling the modeling of the heart (Sainte-Marie et al 2006; Chapelle et al 2012). We also refer to (Peskin 1975) for a variant of the Huxley'57 equations, with a view to a comprehensive description of the cardiac behavior.

Moreover, experimental validations have been obtained for this same model (Hill et al 1975; Zahalak and Ma 1990; Lecarpentier et al 1998; Caruel et al 2014), in addition to detailed validations achieved with clinical data for heart models representing physiological or pathological conditions (Chabiniok et al 2012).

However, the above-noted shortcoming of the Huxley'57 type models regarding the absence of power stroke is not without consequence, as has been observed for some decades, including by Huxley himself with coworkers (Huxley and Simmons 1971). In particular, these models cannot account for the transient behaviors associated with time scales smaller than the time constant of a power stroke - i.e. about $1 \mathrm{~ms}$. Such phenomena are displayed e.g. when submitting pre-activated muscle fibers to very fast load steps by prescribing a sudden change in applied force or extension (Ford et al 1977; Piazzesi et al 2002). This is why a model with two possible chemical states - corresponding to the pre- and post- power stroke configurations of the myosin head engaged in a cross-bridge - was proposed in (Huxley and Simmons 1971), combined with the same elastic component as before to represent the deformations of the cross-bridge in each state. While physical considerations have shown that such a model with only two chemical states and an independent elastic element cannot have the right time constant for the power stroke - leading to the introduction of a number of necessary intermediate chemical states (Smith et al 2008; Smith and Mijailovich 2008; Caremani et al 2015) - Eisenberg and Hill have proposed a theory by which two chemical states interact with each other, and with one mechanical degree-of-freedom (dof) embedded in each chemical state (Eisenberg and Hill 1978). The free energy associated with each state is then parametrized by this mechanical dof (Hill 2004), but - unlike in the approach initiated in Huxley-Simmons'71 - it is not written as the sum of a purely chemical potential (independent of the dof) and of a mechanical strain energy. This means that the energy landscape is much less constrained, in particular for adjusting the energy barriers associated with the transitions between the two states for any given value of the mechanical dof.

An alternative path was proposed in (Marcucci and Truskinovsky 2010a; Caruel et al 2013), where an ensemble of discrete configurations are interpreted as local minima of a rugged multidimensional continuous energy landscape. The dynamics of the system is then modeled by a Langevin-type stochastic evolution of an internal mechanical dof on this landscape, rather than by a discrete jump process with prescribed transition functions. Figure 1 displays the corresponding two-dofs model of a myosin head, with $X^{t}$ denoting the overall extension and $Y^{t}$ the internal dof, and with the internal energy landscape visualized in Fig. 1(b). The necessity of defining a large set of rate functions is then replaced by an adequate parametrization of the landscape. This purely mechanical approach was first used to model the power-stroke-type transition (Marcucci and Truskinovsky 2010a; Caruel et al 2013), and also extended to the active ATP-linked attachment-detachment process by adding a correlated (colored) noise to the un- 
correlated thermal fluctuations of the Langevin equation (Marcucci and Truskinovsky 2010b; Sheshka and Truskinovsky 2014), see also (Caruel and Truskinovsky 2017) for a review.

In this work, we propose to follow an intermediate strategy by retaining the two-dofs model of (Marcucci and Truskinovsky 2010a; Caruel et al 2013) of a myosin head with the bi-stable component accounting for the power stroke mechanism, albeit while using the mechano-chemical formalism of (Hill 2004) to couple this model with the chemistry of binding-unbinding phenomena. On the one hand, the motivation of mechanochemical coupling at this level is very straightforward, as it naturally provides a connection with electrophysiology (calcium) and with energy inputs (ATP), while allowing to use well-established chemical rates for which calibrated values can be found in the literature. On the other hand, the case for a mechanical vision of the myosin head with two dofs - rather than two (or more) different chemical states - mainly lies in the ability to account for two significantly different values of stiffness parameters experimentally measured for different time constants, namely, before and after power stroke can occur. We point out that a similar strategy was recently proposed in (Marcucci et al 2016), see also (Washio et al 2018). Beyond a few technical differences in the microscopic modeling assumptions - three local minima for the power stroke dof in (Marcucci et al 2016) instead of two in our case, and chemical transition within the unbound state instead of mechanical - our main fundamental contribution lies in carrying over the agentbased Langevin dynamics to Fokker-Planck type equations describing the evolution of probability densities. Therefore, we are able to describe the behavior of the overall population of myosin heads, which thus represents a macroscopic model of active contraction in striated muscles. This has major advantages, and in particular it allows us to derive a macroscopic energy balance equation. Furthermore, at this macroscopic level we can rather directly compare our model with previous mechano-chemical models. In particular, we are able to retrieve the Huxley'57 model equations under two types of simplifying assumptions - which provides Langevin counterparts to these historical equations, a novel contribution in itself to our best knowledge: (1) by simply canceling the mechanical dof associated with the power stroke, but also (2) by assuming that the time constant of the macroscopic behavior in consideration is long enough for this internal dof to be at thermal equilibrium within the population. In addition, as we demonstrate in this paper, this thermal equilibrium model proves to be instrumental in order to carefully calibrate the modeling components compatible with the underlying time constant assumption, before fine-tuning the specific parts relevant in faster phenomena.

We emphasize that this paper is focused on the mechanics of myosin heads engaging in cross-bridges, and on the resulting behavior at the sarcomere level. In particular, we consider actin filaments to be fully activated by calcium, i.e. we do not address the kinetics of force generation associated with calcium activation, which has been quite abundantly discussed - see e.g. (Hunter et al 1998) and references therein - although some open questions are still debated by physiologists (de Tombe et al 2010; Sequeira and Velden 2017). Likewise, we do not consider length-dependence effects in the sarcomere, which in cardiac physiology result in the well-known Frank-Starling mechanism, see (Chapelle et al 2012) for a strategy to incorporate this effect in a Huxley'57 type model. All this is justified by the facts that these effects can be incorporated separately, and that physiologists are able to perform experiments in which these effects are discarded, see e.g. (Caremani et al 2016).

The outline of the paper is as follows. In the next section, we introduce our proposed model, first in the form of Langevin stochastic equations describing the dynamics for the dofs of a myosin head, with jumps associated with binding-unbinding chemical reactions. This is followed by a derivation of Fokker-Planck equations governing the related probability density functions associated with the same dofs and chemical states. Then, in Section 3 we use these Fokker-Planck equations to address thermodynamics considerations, i.e. we write the equation for the evolution of free energy including the mechanical contributions - and discuss how the chemical rates should be chosen to satisfy the second principle. Next, in Section 4 we show how our model can be transformed based on several simplifying assumptions, which allows a detailed comparison with models of the Huxley'57 type, in particular. The following Section 5 addresses the detailed calibration of the various model components, based on experimental data available in the literature, and provides some detailed simulation results of the model. Then Section 6 discusses various questions and findings arising from the results. Finally we provide some concluding remarks in Section 7.

\section{Stochastic model}

Our model of myosin heads in sarcomeres is formulated within the classical assumption of rigid sliding filaments, in which myosin heads anchored to so-called thick filaments can bind to specific actin sites located on the thin filaments, see e.g. (Tortora and Derrikson 
2009) for a physiological description, and the pioneering paper (Huxley 1957) formalizing the Huxley'57 model in this framework. In our case, we will increase the modeling complexity - compared to Huxley'57 - by introducing an additional mechanical dof to incorporate the power stroke phenomenon.

\subsection{Model of myosin II}
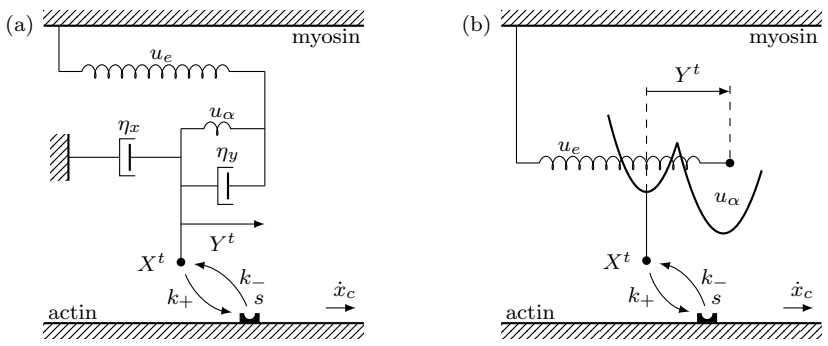

Fig. 1 Kinematical and mechanical model of an individual myosin head, characterized by the position of the tip $X^{t}$ and the internal conformational variable $Y^{t}$ used to account for the power stroke. (a) Schematic representing all the mechanical elements of the cross-bridge rheology. (b) Simplified schematic omitting the damping elements and showing the double-well energy landscape $u_{\alpha}$ associated with the power stroke.

We consider a single myosin head anchored to the thick filament. The variables describing the myosin head are (see Fig. 1):

$-s$ : location of the nearest binding site (relative to myosin);

- $X^{t}$ : location of the head tip $\left(X^{t}=s\right.$ when the head is attached to the binding site);

- $Y^{t}$ : internal degree of freedom associated with the angular orientation of the head with respect to the filament axis;

$-\alpha^{t}$ : attachment state, i.e. a discrete variable that may take one of the two values $\alpha^{t}=1$ when the head is attached to the binding site, and $\alpha^{t}=0$ otherwise.

The actual values of these variables at any given time $t$ will be denoted with $t$ as a superscript.

We assume the actin and myosin filaments to be rigid, so that $\dot{s}=\dot{x}_{c}$, which represents the relative velocity of the actin filament with respect to the myosin filament. The quantity $Y^{t}$ represents the internal degree of freedom of the molecular motors that, in the case of myosin II, accounts for the conformational change responsible for the power stroke (Howard 2001). With these notations, the head switches from pre- to postpower stroke when $Y^{t}$ increases, and the extension of the base component - directly anchored to the myosin filament - of the head is given by $X^{t}+Y^{t}$. We assume - as in (Huxley and Simmons 1971) - this base component to be modeled by a quadratic energy $u_{e}$ (linear spring), while the power stroke component associated with $Y^{t}$ is modeled by a bistable element whose energy is denoted by $u_{\alpha}$ to allow for different expressions according to whether the head is attached or detached, see Fig. 1. Note that both parts of this figure represent our two-dof model of a myosin head, albeit Fig. 1(b) is intended to be more easily interpretable with the representation of the bistable potential, whereas it does not allow the visualization of the damping components. The corresponding total energy landscape is then a function of $\left(X^{t}, Y^{t}\right)$, and we denote this function by $w_{\alpha}(x, y)=u_{\alpha}(y)+u_{e}(x+y)$.

The attachment-detachment process can be viewed as a jump process associated with the discrete variable $\alpha^{t}$. We introduce the rate functions $k_{+}$and $k_{-}$that represent the probability of switching from detached to attached state and vice-versa, per unit of time. We consider that the attachment function depends on the values of $X^{t}$ and $Y^{t}$ before the event and on the position of the nearest binding site $s(t)$. As for the detachment rate, it only depends on $Y^{t}$ and $s(t)$, since $X^{t}=s(t)$ when the head is attached. When an attachment occurs, the value of the variable $X^{t}$ must be adjusted to ensure $X^{t}=s(t)$ after the switch. We consider that, during an attachment event, $X^{t}$ jumps instantaneously from its previous (unattached) position to the position of the binding site $s(t)$, whereas the orientation of the head parametrized by $Y^{t}$ does not change. By contrast, we suppose that a detachment event occurs without changing the values of $X^{t}$ and $Y^{t}$.

Note that, in our model, the power stroke is not associated with a change of chemical states, but is instead modeled as a purely mechanical phenomenon (Marcucci and Truskinovsky 2010a). For consistency, the power stroke recocking is also represented by a purely mechanical event.

In this framework, the dynamics of the mechanical variables are given by the following stochastic differential equations (SDEs) of Langevin type

$$
\left\{\begin{array}{c}
\eta_{x} d X^{t}=\left[\alpha^{t} \eta_{x} \dot{x}_{c}-\left(1-\alpha^{t}\right) \partial_{x} w_{\alpha}\left(X^{t}, Y^{t}\right)\right] d t \\
+\eta_{x} \delta\left(t-t_{s}\right)\left[s(t)-X^{t}\right] d t \\
+\left(1-\alpha^{t}\right) \sqrt{2 \eta_{x} k_{B} T} d B_{x}^{t} \\
\eta_{y} d Y^{t}=\left[-\partial_{y} w_{\alpha}\left(X^{t}, Y^{t}\right)\right] d t+\sqrt{2 \eta_{y} k_{B} T} d B_{y}^{t}
\end{array}\right.
$$

where $\eta_{x}$ and $\eta_{y}$ are the drag coefficients representing the viscous damping due to the surrounding fluid, while $d B_{x}^{t}$ and $d B_{y}^{t}$ are independent increments of two Wiener processes characterized by $\mathbb{E}\left(d B_{x}^{t}\right)=\mathbb{E}\left(d B_{y}^{t}\right)=0$ and 
$\operatorname{Var}\left(d B_{x}^{t}\right)=\operatorname{Var}\left(d B_{y}^{t}\right)=d t, k_{B}$ denotes the Boltzmann constant, and $T$ is the absolute temperature. Note that the value of $\alpha^{t}$ at any time $t$ can only be 0 or 1 , hence the nature of (1a) is drastically different according to the chemical state. In the attached state $\left(\alpha^{t}=1\right)$, it simplifies into $d X^{t}=\dot{x}_{c} d t$ - meaning that the myosin head is constrained to follow the actin filament - whereas we have an actual stochastic dynamical equation when $\alpha^{t}=0$. The term containing the Dirac delta function accounts for a jump at time $t_{s}$, where the degree of free$\operatorname{dom} X^{t}$ will instantaneously switch at $t=t_{s}$ from its value before attachment denoted by $X^{t_{s}}$ to $s\left(t_{s}\right)$. Note indeed that time integration of (1a) between times $t_{s}$ - just before the jump - and $t_{+}$- after the jump and while the head remains attached - gives

$X^{t_{+}}=X^{t_{s}}+\left(s\left(t_{s}\right)-X^{t_{s}}\right)+\dot{x}_{c}\left(t_{+}-t_{s}\right)=s\left(t_{+}\right)$.

For simplicity we will assume that $\eta_{x}=\eta_{y}=\eta$. Introducing the quantity

$D=\frac{k_{B} T}{\eta}$

which can be interpreted as a diffusion coefficient according to Einstein's law of diffusion, we can rewrite System (1) as

$$
\left\{\begin{array}{c}
d X^{t}=\left[\alpha^{t} \dot{x}_{c}-\left(1-\alpha^{t}\right) \eta^{-1} \partial_{x} w_{\alpha}\left(X^{t}, Y^{t}\right)\right] d t \\
+\delta\left(t-t_{s}\right)\left[s(t)-X^{t}\right] d t \\
+\left(1-\alpha^{t}\right) \sqrt{2 D} d B_{x}^{t} \\
d Y^{t}=\left[-\eta^{-1} \partial_{y} w_{\alpha}\left(X^{t}, Y^{t}\right)\right] d t+\sqrt{2 D} d B_{y}^{t}
\end{array}\right.
$$

In addition to the Langevin equations (3), we define the probabilities of transitions between the attached and detached states,

$\left\{\begin{array}{l}\mathbb{P}\left[\alpha^{t+d t}=1 \mid \alpha^{t}=0\right]=k_{+}\left(X^{t}, Y^{t}, s, t\right) d t \\ \mathbb{P}\left[\alpha^{t+d t}=0 \mid \alpha^{t}=1\right]=k_{-}\left(Y^{t}, s, t\right) d t\end{array}\right.$

\subsection{Mesoscale model}

We refer here to the mesoscale as a scale at which the population of myosin heads is sufficiently large for its effects to be satisfactorily represented by a statistical description, namely, typically the myofibril scale where the density of molecular motors is about $300 \times 10^{3}$ units per half-sarcomere (Reconditi 2006; Alberts et al 2014).

We define, for the population considered:

- $P_{\alpha}(s, t)$ the probability for a head located at distance $s$ of the nearest binding site at time $t$ to be in the attached/detached state, with the following normalization condition

$$
P_{1}(s, t)+P_{0}(s, t)=1 \text {. }
$$

- $p(x, y, \alpha ; s, t) d x d y$ the probability for a head - again, located at distance $s$ of the nearest binding site at time $t$ - to be in the state $\alpha$, and between $x$ and $x+d x$ and between $y$ and $y+d y$.

We emphasize that all the previous densities must be understood as quantities pertaining to heads located at a given distance $s$ of the binding site, i.e. $s$ plays the role of a deterministic variable - like time $t$ - not a probabilistic one like $x$ and $y$.

Here, we have the following normalization condition

$\sum_{\alpha=0,1} \iint p(x, y, \alpha ; s, t) d x d y=1$,

and the relation between $P_{\alpha}$ and $p$

$P_{\alpha}(s, t)=\iint p(x, y, \alpha ; s, t) d x d y$.

Moreover, when a head is attached we have $X^{t}=s$, so that we can write a more specific expression for $p$ in the attached state

$p(x, y, 1 ; s, t)=\delta(x-s) \bar{p}(y ; s, t)$.

Note that with this definition $\bar{p}$ is not - strictly speaking - a probability density since $\int \bar{p}(y ; s, t) d y=P_{1}(s, t) \neq$ 1.

Our goal is now to derive a set of equations describing the evolution of these probability densities - namely, so-called Fokker-Planck equations - based on the SDEs of Section 2.1. To that purpose we follow a classical path for this type of statistical mechanics model - see e.g. (Le Bris and Lelièvre 2009, Section 4.1) for more detail - with in our case the addition of jump terms. Let $\phi(x, y, \alpha)$ be a test function, on which we will apply the Itô formula on the stochastic trajectory. For a particle initially in state $\alpha^{t}=0$ - at the beginning of a time increment $d t$ - the variation of $\phi\left(X^{t}, Y^{t}, \alpha^{t}\right)$ gives

$$
\begin{aligned}
\left.d \phi\right|_{\alpha^{t}=0}= & {\left[-\eta^{-1} \partial_{x} w_{0}\left(X^{t}, Y^{t}\right) \partial_{x} \phi\left(X^{t}, Y^{t}, 0\right)\right.} \\
& -\eta^{-1} \partial_{y} w_{0}\left(X^{t}, Y^{t}\right) \partial_{y} \phi\left(X^{t}, Y^{t}, 0\right) \\
& \left.+D \partial_{x}^{2} \phi\left(X^{t}, Y^{t}, 0\right)+D \partial_{y}^{2} \phi\left(X^{t}, Y^{t}, 0\right)\right] d t \\
+ & \left.\phi \phi\left(s, Y^{t}, 1\right)-\phi\left(X^{t}, Y^{t}, 0\right)\right] \delta\left(t-t_{s}\right) d t \\
+ & \sqrt{2 D} \partial_{x} \phi\left(X^{t}, Y^{t}, 0\right) d B_{x}^{t} \\
+ & \sqrt{2 D} \partial_{y} \phi\left(X^{t}, Y^{t}, 0\right) d B_{y}^{t},
\end{aligned}
$$

where we have introduced the fact that during a binding event (jump to state 1) $X^{t}$ switches to $s$ instantaneously at time $t_{s}$.

As for a particle initially in state 1 , we have to take into account that, since $X^{t}=s$, the stochastic part of 
the evolution of $X^{t}$ is cancelled and $d X^{t}=\dot{x}_{c} d t$, see (3). In addition, the jump to state 0 - if any - occurs at fixed $X$ and $Y$. Therefore, the variation of $\phi\left(X^{t}, Y^{t}, \alpha^{t}\right)$ reads

$$
\begin{aligned}
\left.d \phi\right|_{\alpha^{t}=1}=\left[\dot{x}_{c} \partial_{x} \phi\left(X^{t}, Y^{t}, 1\right)\right. & \\
& -\eta^{-1} \partial_{y} w_{1}\left(X^{t}, Y^{t}\right) \partial_{y} \phi\left(X^{t}, Y^{t}, 1\right) \\
& \left.+D \partial_{y}^{2} \phi\left(X^{t}, Y^{t}, 0\right)\right] d t \\
+ & {\left[\phi\left(X^{t}, Y^{t}, 0\right)-\phi\left(X^{t}, Y^{t}, 1\right)\right] \delta\left(t-t_{s}\right) d t } \\
+ & \sqrt{2 D} \partial_{y} \phi\left(X^{t}, Y^{t}, 0\right) d B_{y}^{t} .
\end{aligned}
$$

The expectation variation is then computed by summing the contributions from the 2 types of trajectories and integrating over $x$ and $y$ against the probability density $p$ defined above. We obtain, after integrating by parts and dividing by $d t$,

$$
\begin{gathered}
\frac{d}{d t} \mathbb{E} \phi\left(X^{t}, Y^{t}, \alpha^{t}\right) \\
=\iint \phi(x, y, 0)\left[\partial _ { x } \left(\eta^{-1} \partial_{x} w_{0}(x, y) p(x, y, 0 ; s, t)\right.\right. \\
\left.+D \partial_{x} p(x, y, 0 ; s, t)\right) \\
+\partial_{y}\left(\eta^{-1} \partial_{y} w_{0}(x, y) p(x, y, 0 ; s, t)\right. \\
\left.\left.+D \partial_{y} p(x, y, 0 ; s, t)\right)\right] d x d y \\
+\iint(\phi(s, y, 1)-\phi(x, y, 0)) \\
k_{+}(x, y, s) p(x, y, 0 ; s, t) d x d y \\
+\iint \phi(x, y, 1)\left[\partial_{x}\left(-\dot{x}_{c} p(x, y, 1 ; s, t)\right)\right. \\
+\partial_{y}\left(\eta^{-1} \partial_{y} w_{1}(x, y) p(x, y, 1 ; s, t)\right. \\
\left.\left.+D \partial_{y} p(x, y, 1 ; s, t)\right)\right] d x d y \\
+\iint(\phi(x, y, 0)-\phi(x, y, 1)) \\
k-(y, s) p(x, y, 1 ; s, t) d x d y
\end{gathered}
$$

On the other hand, the expectation is also obtained by direct integration of the density, i.e.

$$
\begin{aligned}
& \mathbb{E} \phi\left(X^{t}, Y^{t}, \alpha^{t}\right) \\
&=\sum_{\alpha=0,1}\left\{\iint \phi(x, y, \alpha) p(x, y, \alpha ; s, t) d x d y\right\}
\end{aligned}
$$

which yields by time-differentiating

$$
\begin{aligned}
& \frac{d}{d t} \mathbb{E} \phi\left(X^{t}, Y^{t}, \alpha^{t}\right) \\
& \quad=\sum_{\alpha=0,1}\left\{\iint \phi(x, y, \alpha) \frac{d}{d t} p(x, y, \alpha ; s, t) d x d y\right\},
\end{aligned}
$$

where the material derivative notation $d / d t$ pertains to time variations of quantities when following a population associated with a given value of $s$ at time $t$, i.e.

$\frac{d}{d t} p(x, y, \alpha ; s, t)=\partial_{t} p(x, y, \alpha ; s, t)+\dot{x}_{c} \partial_{s} p(x, y, \alpha ; s, t)$

As the test function is arbitrary, we can identify its coefficients between Eqs. (11) and (13), which yields

$$
\left\{\begin{aligned}
\frac{d}{d t} p(x, y, 0 ; s, t) & \\
= & \partial_{x}\left(\eta^{-1} \partial_{x} w_{0}(x, y) p(x, y, 0 ; s, t)\right. \\
& \left.+D \partial_{x} p(x, y, 0 ; s, t)\right) \\
& +\partial_{y}\left(\eta^{-1} \partial_{y} w_{0}(x, y) p(x, y, 0 ; s, t)\right. \\
& \left.+D \partial_{y} p(x, y, 0 ; s, t)\right) \\
& +k_{-}(y, s) p(x, y, 1 ; s, t) \\
& -k_{+}(x, y, s) p(x, y, 0 ; s, t) \\
\frac{d}{d t} p(x, y, 1 ; s, t) & \\
= & \partial_{x}\left(-\dot{x}_{c} p(x, y, 1 ; s, t)\right) \\
& +\partial_{y}\left(\eta^{-1} \partial_{y} w_{1}(x, y) p(x, y, 1 ; s, t)\right. \\
& \left.+D \partial_{y} p(x, y, 1 ; s, t)\right) \\
& +\delta(x-s) \int k_{+}(\tilde{x}, y, s) p(\tilde{x}, y, 0 ; s, t) d \tilde{x} \\
& -k_{-}(y, s) p(x, y, 1 ; s, t)
\end{aligned}\right.
$$

where we have used the identity

$$
\begin{aligned}
& \iint \phi(s, y, 1) k_{+}(x, y, s) p(x, y, 0 ; s, t) d x d y= \\
& \int \phi(x, y, 1) \delta(x-s) \iint k_{+}(\tilde{x}, y, s) p(\tilde{x}, y, 0 ; s, t) d \tilde{x} d x d y
\end{aligned}
$$

to deal with the non-standard test function term $\phi(s, y, 1)$ present in (11). Now we further develop the time derivatives, also incorporating (8), into

$$
\left\{\begin{aligned}
& \frac{d}{d t} p(x, y, 0 ; s, t)=\partial_{t} p(x, y, 0 ; s, t) \\
&+ \dot{x}_{c} \partial_{s} p(x, y, 0 ; s, t) \\
& \frac{d}{d t} p(x, y, 1 ; s, t)=\delta(x-s)\left(\partial_{t} \bar{p}(y ; s, t)\right. \\
&\left.+\dot{x}_{c} \partial_{s} \bar{p}(y ; s, t)\right) \\
&-\delta^{\prime}(x-s) \dot{x}_{c} \bar{p}(y ; s, t
\end{aligned}\right.
$$


This leads to our final Fokker-Planck equations

$$
\left\{\begin{aligned}
\partial_{t} p(x, y, 0 ; s, t) & +\dot{x}_{c} \partial_{s} p(x, y, 0 ; s, t) \\
= & \partial_{x}\left(\eta^{-1} \partial_{x} w_{0}(x, y) p(x, y, 0 ; s, t)\right. \\
& \left.+D \partial_{x} p(x, y, 0 ; s, t)\right) \\
& +\partial_{y}\left(\eta^{-1} \partial_{y} w_{0}(x, y) p(x, y, 0 ; s, t)\right. \\
& \left.+D \partial_{y} p(x, y, 0 ; s, t)\right) \\
& +k_{-}(y, s) \delta(x-s) \bar{p}(y ; s, t) \\
& -k_{+}(x, y, s) p(x, y, 0 ; s, t) \\
\partial_{t} \bar{p}(y ; s, t)+ & \dot{x}_{c} \partial_{s} \bar{p}(y ; s, t) \\
= & \partial_{y}\left(\eta^{-1} \partial_{y} w_{1}(s, y) \bar{p}(y ; s, t)\right. \\
& \left.+D \partial_{y} \bar{p}(y ; s, t)\right) \\
& +\int k_{+}(x, y, s) p(x, y, 0 ; s, t) d x \\
& -k_{-}(y, s) \bar{p}(y ; s, t)
\end{aligned}\right.
$$

Note that for $P_{1}(s, t)=\iint p(x, y, 1 ; s, t) d x d y=$ $\int \bar{p}(y ; s, t) d y$ - namely, the overall probability for a head at distance $s$ and time $t$ to be attached - a direct integration of (16b) gives

$$
\begin{aligned}
& \partial_{t} P_{1}(s, t)+\dot{x}_{c} \partial_{s} P_{1}(s, t) \\
& =\iint k_{+}(x, y, s) p(x, y, 0 ; s, t) d x d y \\
& \quad-\int k_{-}(y, s) \bar{p}(y ; s, t) d y .
\end{aligned}
$$

Likewise, for the overall probability of the detached state $P_{0}(s, t)=\iint p(x, y, 0 ; s, t) d x d y$, we have using (16a)

$$
\begin{aligned}
& \partial_{t} P_{0}(s, t)+\dot{x}_{c} \partial_{s} P_{0}(s, t) \\
& =\int k_{-}(y, s) \bar{p}(y ; s, t) d y \\
& \quad-\iint k_{+}(x, y, s) p(x, y, 0 ; s, t) d x d y
\end{aligned}
$$

so that we verify $\frac{d}{d t} P_{1}+\frac{d}{d t} P_{0}=0$ as expected from the normalization identity (5). We can then rewrite (17) as

$$
\begin{aligned}
\partial_{t} P_{1}(s, t) & +\dot{x}_{c} \partial_{s} P_{1}(s, t) \\
& =f(s, t)\left(1-P_{1}(s, t)\right)-g(s, t) P_{1}(s, t),
\end{aligned}
$$

where the attachment and detachment functions $f$ and $g$ are given by

$f(s, t)=\iint k_{+}(x, y, s) \frac{p(x, y, 0 ; s, t)}{1-P_{1}(s, t)} d x d y$,

$g(s, t)=\int k_{-}(y, s) \frac{\bar{p}(y ; s, t)}{P_{1}(s, t)} d y$

and we recognize in (19) the fundamental equation of the Huxley'57 model (Huxley 1957), but here $f$ and $g$ are ad hoc since they depend on $P_{1}$ itself, in particular.

\section{Thermodynamics considerations}

We follow (Hill 2004) and define the chemical potentials of the two states as

$$
\begin{aligned}
\mu_{0}(x, y ; s, t) & =w_{0}(x, y)+k_{B} T \ln [p(x, y, 0 ; s, t)], \\
\mu_{1}(y ; s, t) & =w_{1}(s, y)+k_{B} T \ln [\bar{p}(y ; s, t)] .
\end{aligned}
$$

Note that these potentials incorporate the mechanical energies $w_{\alpha}$ discussed in Section 2.1. The free energy is then given by the overall mean value of chemical potential, i.e.

$$
\begin{aligned}
F(s, t)=\iint \mu_{0}(x, y ; s, t) & p(x, y, 0 ; s, t) d x d y \\
& +\int \mu_{1}(y ; s, t) \bar{p}(y ; s, t) d y .
\end{aligned}
$$

We now proceed to compute the total time derivative of this free energy, namely,

$$
\begin{aligned}
& \frac{d}{d t} F(s, t)=\partial_{t} F(s, t)+\dot{x}_{c} \partial_{s} F(s, t) \\
& =\iint\left(\partial_{t} p(x, y, 0 ; s, t)+\dot{x}_{c} \partial_{s} p(x, y, 0 ; s, t)\right) \\
& \quad \times\left(w_{0}(x, y)+k_{B} T(\ln [p(x, y, 0 ; s, t)]+1)\right) d x d y \\
& +\int\left(\partial_{t} \bar{p}(y ; s, t)+\dot{x}_{c} \partial_{s} \bar{p}(y ; s, t)\right) \\
& \quad\left(w_{1}(s, y)+k_{B} T(\ln [\bar{p}(y ; s, t)]+1)\right) d y \\
& +\dot{x}_{c} \int \partial_{s} w_{1}(s, y) \bar{p}(y ; s, t) d y .
\end{aligned}
$$

We denote

$$
\tau_{c}=\int \partial_{s} w_{1}(s, y) \bar{p}(y ; s, t) d y
$$

which represents by definition the average value of the active tension developed in attached myosin heads. Using the above Fokker-Planck equations (16) and recalling that $k_{B} T=\eta D$, we then obtain after some straightforward manipulations

$$
\begin{aligned}
& \frac{d}{d t} F(s, t)=\tau_{c} \dot{x}_{c} \\
& -\iint \eta^{-1} p(x, y, 0 ; s, t) \\
& \quad \times\left[\left(\partial_{x} \mu_{0}(x, y ; s, t)\right)^{2}+\left(\partial_{y} \mu_{0}(x, y ; s, t)\right)^{2}\right] d x d y \\
& -\int \eta^{-1} \bar{p}(y ; s, t)\left(\partial_{y} \mu_{1}(y ; s, t)\right)^{2} d y \\
& +\iint k_{+}(x, y, s) p(x, y, 0 ; s, t) \\
& \quad \times\left[\mu_{1}(y ; s, t)-\mu_{0}(x, y ; s, t)\right] d x d y \\
& +\int k_{-}(y, s) \bar{p}(y, s)\left[\mu_{0}(s, y ; s, t)-\mu_{1}(y ; s, t)\right] d y .
\end{aligned}
$$


In fact, in order to obtain more insight from this balance, we need to take into account the fact that detachment involves some energy input due to the consumption of one ATP in each reaction. We will here make the simplifying assumption that detachment only occurs within the chemical reaction involving ATP, indeed, see also Section 6 for a related discussion on the fundamental point concerning direct and inverse reactions. Denoting by $\mu_{T}$ the chemical potential of ATP in the solution - note that ATP concentration is kept stable in physiological conditions - and by $\bar{J}_{1}^{0}$ the flux of the detachment reaction, i.e.

$\bar{J}_{1}^{0}=\int k_{-}(y, s) \bar{p}(y ; s, t) d y$,

we then rewrite the equation as

$$
\begin{aligned}
& \frac{d}{d t} F(s, t)=\tau_{c} \dot{x}_{c}+\bar{J}_{1}^{0} \mu_{T} \\
& -\iint \eta^{-1} p(x, y, 0 ; s, t) \\
& \quad \times\left[\left(\partial_{x} \mu_{0}(x, y ; s, t)\right)^{2}+\left(\partial_{y} \mu_{0}(x, y ; s, t)\right)^{2}\right] d x d y \\
& -\int \eta^{-1} \bar{p}(y ; s, t)\left(\partial_{y} \mu_{1}(y ; s, t)\right)^{2} d y \\
& +\iint k_{+}(x, y, s) p(x, y, 0 ; s, t) \\
& \quad \quad \times\left[\mu_{1}(y ; s, t)-\mu_{0}(x, y ; s, t)\right] d x d y \\
& +\int k_{-}(y, s) \bar{p}(y, s) \\
& \quad \times\left[\mu_{0}(s, y ; s, t)-\left(\mu_{1}(y ; s, t)+\mu_{T}\right)\right] d y
\end{aligned}
$$

This is an energy balance equation, which can be interpreted as

$\frac{d}{d t} F(s, t)=\mathcal{P}_{e x t}+\bar{J}_{1}^{0} \mu_{T}-T \dot{S}_{\text {prod }}$,

where $\mathcal{P}_{\text {ext }}=\tau_{c} \dot{x}_{c}$ denotes the power of external forces, $\bar{J}_{1}^{0} \mu_{T}$ the influx of chemical potential provided by ATP, and $\dot{S}_{\text {prod }}$ the entropy production rate given by

$$
\begin{aligned}
\dot{S}_{\text {prod }}=T^{-1}\left[\iint\right. & \eta^{-1} p(x, y, 0 ; s, t)\left[\left(\partial_{x} \mu_{0}(x, y ; s, t)\right)^{2}\right. \\
& \left.+\left(\partial_{y} \mu_{0}(x, y ; s, t)\right)^{2}\right] d x d y \\
+ & \int \eta^{-1} \bar{p}(y ; s, t)\left(\partial_{y} \mu_{1}(y ; s, t)\right)^{2} d y \\
+ & \iint k_{+}(x, y, s) p(x, y, 0 ; s, t) \\
& \times\left[\mu_{0}(x, y ; s, t)-\mu_{1}(y ; s, t)\right] d x d y \\
+ & \int k_{-}(y, s) \bar{p}(y, s) \\
& \left.\times\left[\mu_{1}(y ; s, t)+\mu_{T}-\mu_{0}(s, y ; s, t)\right] d y\right] .
\end{aligned}
$$

Here, as usual, satisfying the second principle of thermodynamics requires reaction rates to be defined so that the last two terms always give a positive contribution (Hill 2004).

We finally point out that all the above quantities and balances, in particular - have been considered for specific values of $s$, i.e. they pertain to heads located at a distance $s$ from their nearest possible binding site. Therefore, in order to obtain the corresponding global quantities we should integrate over all possible values of $s$ - typically, $s \in\left[s_{-}, s_{-}+d_{a}\right]$ where $d_{a}$ denotes the spacing between two adjacent available binding sites on the actin filaments, with a uniform distribution of $s$ over this interval.

\section{Model simplifications and comparisons}

The objective in this section is to investigate models that can be derived from our above-proposed model equations under various simplifying assumptions, in order to place our model in a larger perspective and to allow inter-model comparisons, both in their structures and the results they can provide. In particular, we will see that we can obtain model equations similar to Huxley'57 under two different types of assumptions, associated with the following subsections.

\subsection{Direct simplification of kinematical assumptions}

We consider (19)-(21), and we assume as in Huxley'57 that there is only one degree of freedom in a myosin head - namely, $X^{t}$ - which is equivalent to assuming that $Y^{t}$ has a fixed value $Y^{t}=0$, hence,

$$
\begin{aligned}
\bar{p}(y ; s, t) & =P_{1}(s, t) \delta(y), \\
p(x, y, 0 ; s, t) & =\hat{p}(x ; s, t) \delta(y) .
\end{aligned}
$$

We also follow Huxley'57 in assuming that the probability of attachment does not depend on $X^{t}$, i.e.

$k_{+}(x, 0, s)=f(s)$,

which is consistent with (20) since

$$
\begin{aligned}
\iint p(x, y, 0 ; s, t) d x d y=\int \hat{p}(x ; s, t) d x & \\
& =P_{0}(s, t)=1-P_{1}(s, t) .
\end{aligned}
$$

Moreover, (21) yields

$g(s, t)=k_{-}(0, s)=g(s)$,

and we recover the original Huxley equation

$$
\begin{aligned}
\partial_{t} P_{1}(s, t)+\dot{x}_{c} \partial_{s} P_{1}(s, t) \\
\quad=f(s)\left(1-P_{1}(s, t)\right)-g(s) P_{1}(s, t),
\end{aligned}
$$


with $f$ and $g$ creation and destruction rates that now only depend on $s$. Note that, although this result was to be expected, this derivation shows that there is a Langevin form for the Huxley'57 model.

\subsection{Thermal equilibrium model}

When considering phenomena associated with time constants over which thermal equilibrium can be assumed to hold for the mechanical degrees of freedom - namely, typically larger than $10 \mathrm{~ms}$ (Hill 1974, 1976) - the probability density functions can be computed based on the canonical distributions associated with the energy landscape in each state. Namely, we then have

$$
\begin{aligned}
p(x, y, 0 ; s, t) & =p_{0}^{t h}(x, y) P_{0}(s, t), \\
\bar{p}(y ; s, t) & =p_{1}^{t h}(y ; s) P_{1}(s, t),
\end{aligned}
$$

with

$p_{0}^{t h}(x, y)=Z_{0}^{-1} \exp \left[-\frac{w_{0}(x, y)}{k_{B} T}\right]$,

$p_{1}^{t h}(y ; s)=Z_{1}(s)^{-1} \exp \left[-\frac{w_{1}(s, y)}{k_{B} T}\right]$,

where the partition functions $Z_{0}$ and $Z_{1}(s)$ are given by

$$
\begin{aligned}
& Z_{0}=\iint \exp \left[-\frac{w_{0}(x, y)}{k_{B} T}\right] d x d y \\
& Z_{1}(s)=\int \exp \left[-\frac{w_{1}(s, y)}{k_{B} T}\right] d y .
\end{aligned}
$$

In this case, (20) and (21) simplify into

$$
\begin{aligned}
f^{t h}(s) & =\iint k_{+}(x, y, s) p_{0}^{t h}(x, y) d x d y \\
g^{t h}(s) & =\int k_{-}(y, s) p_{1}^{t h}(y ; s) d y
\end{aligned}
$$

and this provides a full justification of the Huxley equation (19) with reaction rates $(f, g)=\left(f^{t h}, g^{\text {th }}\right)$ now independent of the ratio of currently attached bridges $P_{1}(s, t)$. Note that a similar simplification has also been considered in the case of discrete attached states in (Zahalak 2000). We emphasize that our justification holds here in the framework of more complex modeling assumptions than in Huxley'57, since in our case myosin heads are associated with two mechanical degrees of freedom. In the rest of this paper, we will call the simplified model obtained here the thermal equilibrium model, by which we mean that the degrees of freedom associated with the myosin heads are distributed according to the probabilities (34) in each of the attachment states.
4.3 Derivation of macroscopic model equations

It is very valuable - to reduce complexity and gain insight - to obtain completely macroscopic equations describing the muscle behavior, i.e. relations governing macroscopic physical quantities such as stresses and strains. We now show how this can be achieved starting from Huxley'57 equations, and we will use the results as comparisons in our interpretations and discussions of our stochastic model below. We assume here that the deformations of all the half-sarcomeres constituting a myofibril are identical, see e.g. (Givli 2010) and references therein for modeling the effect of local inhomogeneities of sarcomeres in muscle contraction.

As proposed in (Zahalak 1981), we can introduce the moment quantities

$M_{p}(t)=\frac{1}{d_{a}} \int_{s=s_{-}}^{s_{-}+d_{a}} s^{p} P_{1}(s, t) d s$,

with $d_{a}$ the spacing between two adjacent available binding sites on the actin filaments. Note that it is reasonable to assume that the span of myosin heads is much smaller than $d_{a}$, hence $P_{1}(s, t)$ tends to zero when $s$ tends to the ends of the interval $\left[s_{-}, s_{-}+d_{a}\right]$. We point out that $M_{0}$ represents the overall ratio of actually created cross-bridges among all myosin heads, while $M_{1} / M_{0}$ is the average extension (value of $X=s$ ) in the active cross-bridges. Then we infer from (32), for $p \geq 1$ and using an integration by parts,

$$
\begin{aligned}
\dot{M}_{p}=p & \dot{x}_{c} M_{p-1}+f_{p} \\
- & \frac{1}{d_{a}} \int_{s=s_{-}}^{s_{-}+d_{a}}(f(s)+g(s)) s^{p} P_{1}(s, t) d s,
\end{aligned}
$$

with

$f_{p}(s)=\frac{1}{d_{a}} \int_{s=s_{-}}^{s_{-}+d_{a}} s^{p} f(s) d s$.

In order to use this sequence of dynamical equations to solve for the moments $M_{p}$, the main difficulty consists in dealing with the integral term that still contains $P_{1}(s, t)$, as the factor $f(s)+g(s)$ may in essence involve a large number of monomials $s^{q}$ - leading to coupling with many moments - for a correct representation. Various approaches have been proposed to tackle this problem, in particular by considering specific shapes of the function $P_{1}(s, t)$ (Zahalak 1981).

Now, if we assume as in (Bestel et al 2001; Guérin et al 2011; Chapelle et al 2012) that the combination $f(s)+g(s)$ does not depend on $s$, but only on quantities that are homogeneous at the sarcomere level - such as 
$x_{c}$ or various chemical concentrations - we have the simplification

$\dot{M}_{p}=p \dot{x}_{c} M_{p-1}-(f+g) M_{p}+f_{p}$.

For $p=0$ we directly have

$\dot{M}_{0}=-(f+g) M_{0}+f_{0}$.

Furthermore, a straightforward derivation of the active force can be obtained when considering as in (Bestel et al 2001; Chapelle et al 2012) a quadratic internal elastic energy of the form (attached state)

$w_{1}(x)=w_{e}(x)=\frac{\kappa_{m}}{2}\left(x+s_{0}\right)^{2}$,

where $\kappa_{m}$ denotes the individual stiffness of the myosin head, and $-s_{0}$ represents the equilibrium position of this linear spring. Then the tension generated in the tissue per unit area of fiber cross-section (in the reference configuration) is given by the sum of the individual myosin contributions, that we can compute e.g. by considering a half-sarcomere - of length $\ell_{h s}$ in the reference configuration - as

$T_{c}=\frac{1}{d_{a}} \int_{s=s_{-}}^{s_{-}+d_{a}} \rho_{m} \ell_{h s} \kappa_{m}\left(s+s_{0}\right) P_{1}(s, t) d s$,

where $\rho_{m}$ denotes the number of myosin heads per unit volume in the reference configuration, and therefore

$T_{c}=\ell_{h s} k_{v o l}\left(s_{0} M_{0}+M_{1}\right)$

with $k_{v o l}=\rho_{m} \kappa_{m}$. Defining now the macroscopic stiffness quantity

$K_{c}=\ell_{h s}^{2} k_{v o l} M_{0}$,

the direct application of (38)-(39) gives

$\left\{\begin{array}{l}\dot{K}_{c}=-(f+g) K_{c}+f_{0} K_{\infty} \\ \dot{T}_{c}=-(f+g) T_{c}+K_{c} \dot{e}_{c}+\frac{s_{0} f_{0}+f_{1}}{\ell_{h s}} K_{\infty}\end{array}\right.$

with $K_{\infty}=\ell_{h s}^{2} k_{v o l}$ and $\dot{e}_{c}=\dot{x}_{c} / \ell_{h s}$. Note that $K_{\infty}$ corresponds to the (virtual) tangent stiffness that would be associated with all cross-bridges attached at the same time. This type of model has the major advantage that only the above system of ordinary differential equations needs to be solved to fully describe the macroscopic mechanical behavior, not the partial differential equation governing $P_{1}(s, t)$.

In isometric condition, the steady state stiffness and tension are then given by

$\check{K}_{c}=\frac{f_{0} K_{\infty}}{f+g}, \quad \check{T}_{c}=\frac{s_{0}+f_{1} / f_{0}}{\ell_{h s}} \check{K}_{c}$.
One crucial capability of striated muscles is also that they must be able to sustain significant deformation rates, and in particular substantial shortening rates without drastic drop in the active tension due to the tangent stiffness of attached cross-bridges. One should note - indeed - that there are competing effects in this stiffnessrelated tension variation versus the ability of crossbridges to detach and reattach at a different location to reinforce active tension. In order to illustrate this, we can directly infer from (41a)-(41b) the steady-state active tension obtained under constant strain rate as

$T_{c}=\check{T}_{c}+\frac{\check{K}_{c}}{f+g} \dot{e}_{c}$

Therefore, the efficiency is maximized in shortening when the tangent stiffness $K_{0}$ is low and the reaction rates are high. This also shows that this type of model can be quite directly calibrated based on measurements of the isometric tension, and of the variations of the active tension in sliding - such as in force-velocity curves. We will further discuss stiffness considerations in the final discussion section.

\section{Model calibration and results}

In this section we demonstrate how the model can be consistently calibrated using published values of some directly measured parameters, and data from single fiber experiments. Note that we will only consider situations in which we can neglect the purely passive contribution compared to the active force in the overall measured tension. For calibration purposes we will abundantly use as an intermediate step the simplification of our model introduced in Section 4.2 - i.e. the thermal equilibrium model - before performing complete simulations of our stochastic model for validation.

\subsection{Physical requirements}

The calibration procedure of these parameters is explained in the next subsections, except when the values are taken directly from the existing literature. We will start with the mechanical parameters (energy landscapes), before considering the reaction rates.

\subsubsection{Energy landscapes}

The energy landscape associated with the power stroke is modeled by a bistable potential, which we represent by the piecewise parabolic functions $u_{\alpha}(y)-$ for $\alpha=0$ or 1 - shown in Fig. 2(a) as proposed in (Marcucci and Truskinovsky 2010a) for the attached state, see 
Table 1 Parameters of the model calibrated using data from single fiber experiments found in (Edman and Hwang 1977; Ford et al 1977; Linari et al 2009)

\begin{tabular}{|c|c|c|c|}
\hline Parameter & Symbol & Value & Unit \\
\hline \multicolumn{4}{|l|}{ Power stroke potentials } \\
\hline $\begin{array}{l}\text { Power stroke characteristic length } \\
\text { Bistable element in attached state }(\alpha=1)\end{array}$ & a & 11 & $\mathrm{~nm}$ \\
\hline $\bar{u}_{1}(y)= \begin{cases}\left(\kappa_{1 \text { pre }} / 2\right) y^{2}+v_{1} & \text { if } y \leq \ell_{1} \\
\left(\kappa_{1 \text { post }} / 2\right)(y-a)^{2} & \text { otherwize }\end{cases}$ & $\begin{array}{c}\kappa_{1 \text { pre }} \\
\kappa_{1 \text { post }} \\
\ell_{1}\end{array}$ & $\begin{array}{l}2.72 \\
1.54 \\
2.2\end{array}$ & $\begin{array}{l}\mathrm{pN} \mathrm{nm}^{-1} \\
\mathrm{pN} \mathrm{nm}^{-1} \\
\mathrm{~nm}\end{array}$ \\
\hline$v_{1}=\left(\kappa_{1_{\mathrm{post}}} / 2\right)\left(\ell_{1}-a\right)^{2}-\left(\kappa_{1_{\mathrm{pre}}} / 2\right) \ell_{1}^{2}$ & $v_{1}$ & 53 & $\mathrm{zJ}$ \\
\hline \multicolumn{4}{|l|}{ Bistable element in detached state $(\alpha=0)$} \\
\hline $\begin{array}{l}\bar{u}_{0}(y)= \begin{cases}\left(\kappa_{0 \text { pre }} / 2\right) y^{2}+v_{0} & \text { if } y \leq \ell_{0} \\
\left(\kappa_{0 \text { post }} / 2\right)\left(y-a_{0}\right)^{2} & \text { otherwize }\end{cases} \\
v_{0}=\left(\kappa_{0 \text { post }} / 2\right)\left(\ell_{0}-a_{0}\right)^{2}-\left(\kappa_{0 \text { pre }} / 2\right) \ell_{0}^{2}\end{array}$ & $\begin{array}{c}a_{0} \\
\kappa_{0 \text { pre }} \\
\kappa_{0 \text { post }} \\
\ell_{0} \\
v_{0}\end{array}$ & $\begin{array}{l}7.7 \\
2.72 \\
0.41 \\
3.3 \\
-10.9\end{array}$ & $\begin{array}{l}\mathrm{nm} \\
\mathrm{pN} \mathrm{nm}^{-1} \\
\mathrm{pN} \mathrm{nm}^{-1} \\
\mathrm{~nm} \\
\mathrm{zJ}\end{array}$ \\
\hline \multicolumn{4}{|c|}{ Energy landscapes $w_{\alpha}(x, y)=u_{\alpha}(y)+u_{e}(x+y)($ Fig.2) } \\
\hline $\begin{array}{l}u_{\alpha}(y)=\bar{u}_{\alpha}\left(y+s_{\alpha}\right) \\
u_{e}(x+y)=(\kappa / 2)(x+y)^{2}\end{array}$ & $\begin{array}{c}\kappa \\
s_{0} \\
s_{1}\end{array}$ & $\begin{array}{l}2.72 \\
0 \\
1.9\end{array}$ & $\begin{array}{l}\mathrm{pN} \mathrm{nm}^{-1} \\
\mathrm{~nm} \\
\mathrm{~nm}\end{array}$ \\
\hline \multicolumn{4}{|l|}{ Stochastic dynamics } \\
\hline $\begin{array}{l}\text { Drag coefficient } \\
\text { Microscopic timescale }\end{array}$ & $\begin{array}{c}\eta \\
\gamma=\eta / \kappa\end{array}$ & $\begin{array}{l}0.99 \\
0.36\end{array}$ & $\begin{array}{l}\mathrm{ms} \mathrm{pN} \mathrm{nm}^{-1} \\
\mathrm{~ms}\end{array}$ \\
\hline \multicolumn{4}{|c|}{ Attachment / detachment rates (Figs.3-4) } \\
\hline $\begin{array}{l}k_{+}(s, y)=\psi_{0 \text { pre }}(y) \bar{k}_{+}(s) \\
k_{-}(s, y)=\psi_{1 \text { pre }}(y) \bar{k}_{- \text {pre }}(s, y)+\psi_{1 \text { post }}(y) \bar{k}_{- \text {post }}(s, y) \\
\psi_{\alpha \text { pre }}(y)=1 / 2\left\{1+\tanh \left[\lambda_{1}\left(\ell_{\alpha}-y-s_{\alpha}\right)\right]\right\}, \quad \psi_{\alpha \text { post }}=1-\psi_{0 \alpha} \\
\bar{k}_{+}(s)=k_{1} \exp \left[-\lambda_{2} s^{6}\right] \\
\bar{k}_{- \text {post }}(s, y)=k_{\min }+\left(k_{0} / 2\right)\left\{1-\tanh \left[\lambda_{3}\left(s+y-\ell_{-}\right)\right]\right\} \\
\bar{k}_{- \text {pre }}(s, y)=k_{\min }+k_{1} \exp \left[\lambda_{4}\left(s+y-\ell_{+}\right)\right]\end{array}$ & $\begin{array}{c}\lambda_{1} \\
k_{1} \\
k_{0} \\
\lambda_{2} \\
k_{\min } \\
\lambda_{3} \\
\ell_{+} \\
\lambda_{4} \\
\ell_{-}\end{array}$ & $\begin{array}{l}7.3 \\
48.9 \\
1378 \\
62 \times 10^{-6} \\
11 \\
318 \\
5.0 \\
2.3 \\
-1.7\end{array}$ & $\begin{array}{l}\mathrm{nm}^{-1} \\
\mathrm{~s}^{-1} \\
\mathrm{~nm}^{-6} \\
\mathrm{~s}^{-1} \\
\mathrm{~nm} \\
\mathrm{~nm} \\
\mathrm{~nm} \\
\mathrm{~nm}\end{array}$ \\
\hline \multicolumn{4}{|l|}{ Scaling parameters } \\
\hline $\begin{array}{l}\text { Maximum number of cross-bridges per myosin filament } \\
\text { Reference length of a half sarcomere } \\
\text { Density of thick filament in the muscle cross section } \\
\text { Myofilaments compliance }\end{array}$ & $\begin{array}{c}N \\
\ell_{h s} \\
\rho_{\text {sarc }} \\
C_{m}\end{array}$ & $\begin{array}{l}300 \\
1 \\
5 \times 10^{14} \\
13\end{array}$ & $\begin{array}{l}\mu \mathrm{m} \\
\mathrm{m}^{-2} \\
\mathrm{~nm} \mathrm{MPa}\end{array}$ \\
\hline
\end{tabular}

also their detailed definitions and associated parameters in Table 1. The myosin head is thus characterized by 7 parameters, namely, $a$, the power stroke characteristic length, $\kappa_{\alpha_{\text {pre }}}$ and $\kappa_{\alpha_{\text {post }}}$ the stiffnesses of the pre-and post-power stroke conformations, respectively, and $\ell_{\alpha}$, the position of the energy barriers between the states. Note that the intrinsic energy difference between the pre- and post-power stroke states $v_{\alpha}$ is then constrained to preserve continuity of the energy functional. The existence of the two conformations, which was first postulated in (Huxley and Simmons 1971), was confirmed by X-ray crystallography in (Dominguez et al 1998; Rayment et al 1993). The power stroke was shown to correspond to a rotation of about $70^{\circ}$ of the lever arm present in the myosin head, leading to a displacement of its tip of about $11 \mathrm{~nm}$, hence we associate this value with our characteristic length $a$. The calibration of the other parameters characterizing the energy landscape is explained in Section 5.2.

\subsubsection{Energy of a cross-bridge}

To take into account the intrinsic elasticity of the myosin head, the bistable element is associated in series with an elastic element with energy $u_{e}$ which we consider as linear elastic with the stiffness $\kappa$, so that the overall energy of a single cross-bridge is given by $w_{\alpha}(x, y)=$ 


\section{(a)}
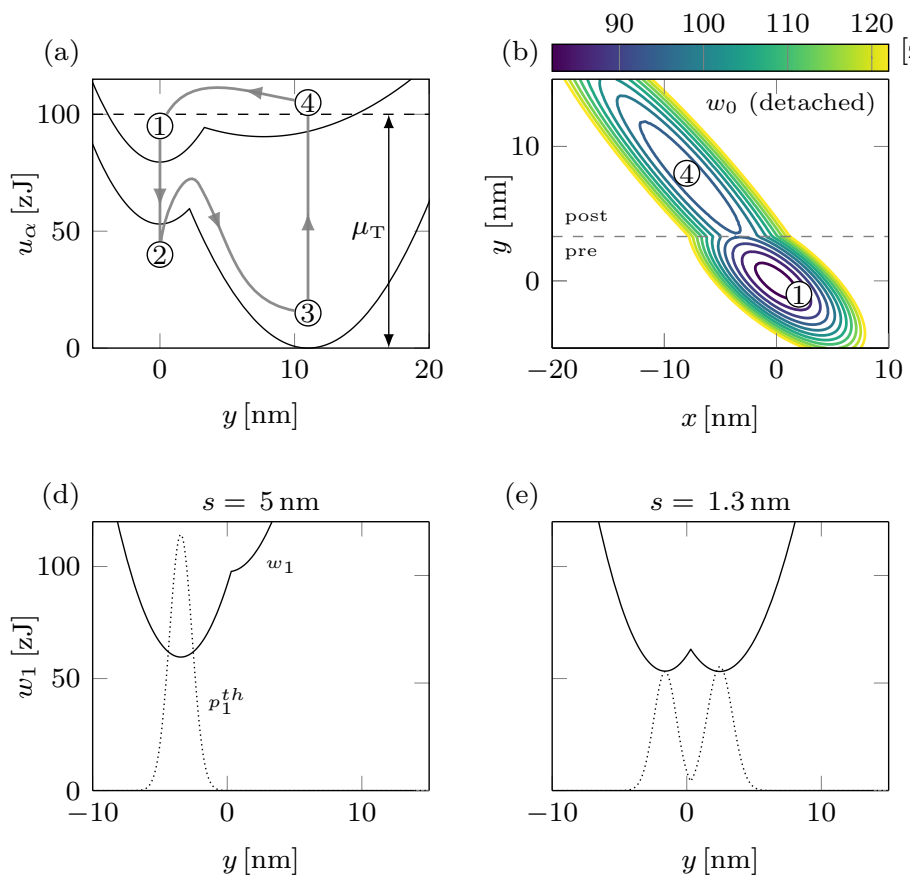

(e)

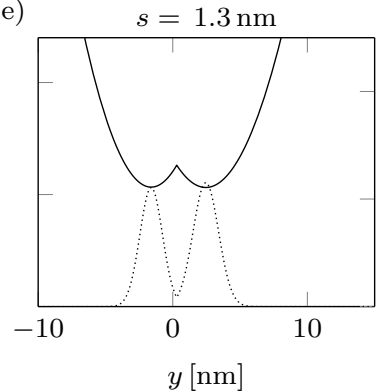

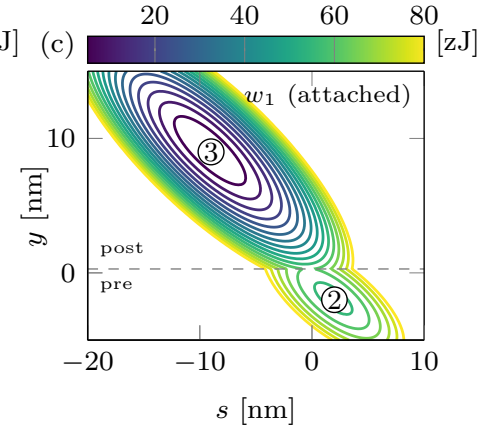

(f)

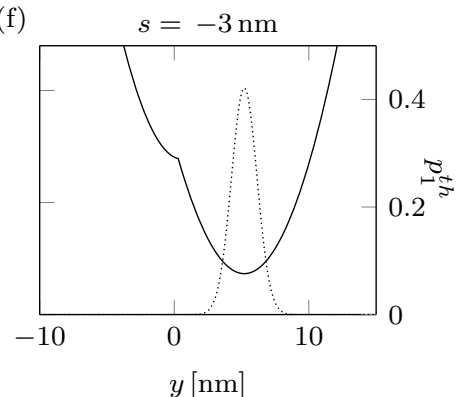

Fig. 2 (a) Representation of the bistable potential in the attached $\left(u_{1}\right)$ and detached $\left(u_{0}\right)$ states; the detached state is shown shifted by the amount of energy provided by ATP hydrolysis $\mu_{T}$ and the gray line represents a typical Lymn-Taylor cycle with four stages (Lymn and Taylor 1971): (1) detached pre-, (2) attached pre- (3) attached post- (4) detached post-power-stroke. (b) and (c) Contours of the overall energy $w_{\alpha}$ in the detached (b) and attached (c) states; the horizontal dashed lines show the position of the energy barrier between the pre- and the post-power stroke states. (d)-(f) overall energy $w_{1}$ (solid line) and equilibrium distribution (dotted line) in the attached state for different values of $s$.

$u_{\alpha}(y)+u_{e}(x+y)=\bar{u}_{\alpha}\left(y+s_{\alpha}\right)+(\kappa / 2)(x+y)^{2}$. Here we introduced a reference length $s_{\alpha}$, which amounts for the attached state to an average pre-strain in isometric conditions, see Section 5.2.2, for the details of the calibration. The level sets of the total cross-bridge energy are represented in Figs. 2(b)-(c). Notice that the energy landscape appears as a function of $x$ and $y$ in the detached state [see (b)], and as a function of $s$ and $y$ in the attached state [see (c)], since $x=s$ when the myosin head is bound to the actin filament, thus forming a cross-bridge. The energy of a cross-bridge $w_{1}$ and the equilibrium distribution $p_{1}^{t h}$ are represented as functions of the power-stroke variable $y$ for different values of $s$ in Figs. 2(d)-(f). At large $s$, the landscape is biased towards the pre-power stroke state [see (d)]. Around $s=0$, both pre- and post-power stroke states are accessible [see (e)], whereas at further shortening only the post-power stroke conformation is populated [see (f)].

\subsubsection{Attachment and detachment rates}

The dynamics of the coordinate $\alpha$, describing attachment and detachment, is controlled by the transition rates $k_{+}$and $k_{-}$, which were introduced in (4). These transition rates are functions of the mechanical degrees of freedom $(x, y)$, and also of $s$, which provides a large leeway of choices. In this paper, we will provide a form by no means unique - fulfilling essential and consensual criteria. One goal of our model is to reproduce the four steps of the Lymn-Taylor cycle, namely, (i) the myosin head attaches in a pre-power stroke position, (ii) the cross-bridge executes the power stroke while attached, (iii) the cross-bridge detaches after the power stroke is completed, in such a way to prevent the motor to be counterproductive, and (iv) the detached head returns to its original pre-power stroke conformation before it can attach again.

One can see that the most basic requirement is that the rates depend on the conformation of the cross-bridge, i.e. on the position of the power stroke variable $y$ with respect to the location of the energy barrier between the states, $\ell_{\alpha}$. Therefore, we introduce the "logic" functions $\psi_{\alpha_{\text {pre }}}(y)$ and $\psi_{\alpha_{\text {post }}}(y)$ shown in Fig. 3(a).

We then prescribe that the attachment can only occur when the myosin head is in the vicinity of an actin attachment site. As the distance between the head and the nearest binding site is given by $s$, we introduce the function $\bar{k}_{+}(s)$ displayed in Fig. 3(b), and we define the attachment rate as $k_{+}(s, y)=\psi_{0 \text { pre }}(y) \bar{k}_{+}(s)$, which implies that the attachment occurs only when the myosin head is detached in its pre-power-stroke conformation 


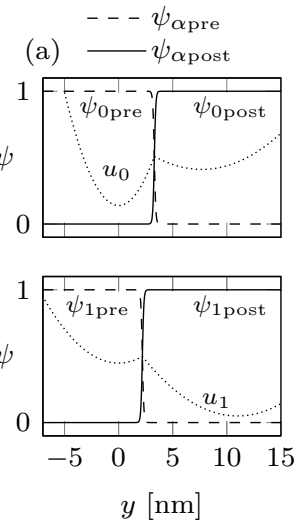

(b)

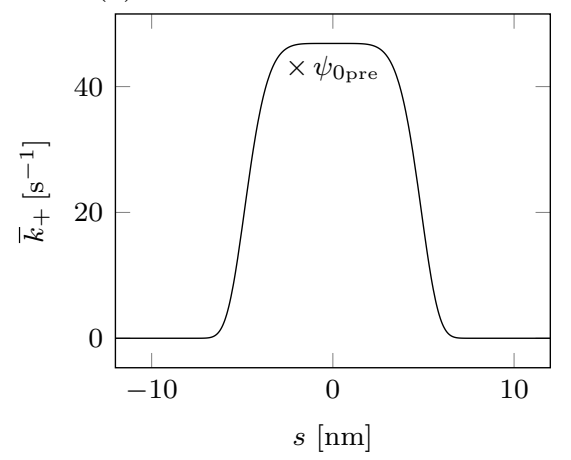

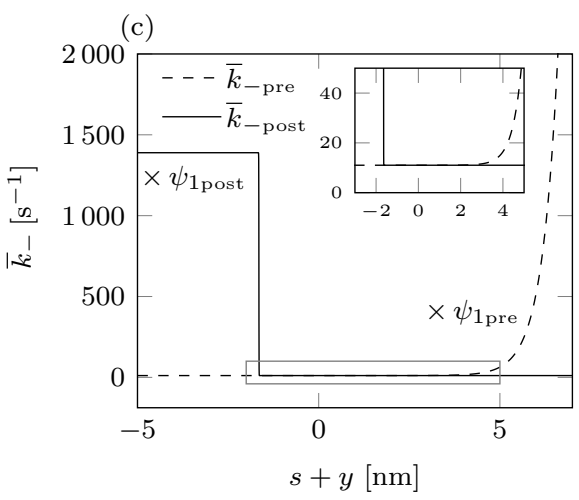

Fig. 3 Attachment and detachment functions. (a) Indicator functions $\psi_{\alpha \text { pre }}$ and $\psi_{\alpha \text { post }}$, designating the pre-and post-power stroke configuration, respectively in the detached (top) and attached (bottom) states. (b) Attachment function from the prepower stroke state. (c) Detachment functions from the pre- (dashed) and the post- (solid) power stroke states with rectangular area zoomed in inset.

(function $\psi_{0_{\text {pre }}}$ ) and sufficiently close to a binding site (function $\bar{k}_{+}$).

The detachment rate function is known to increase with the compressive force borne by the cross-bridge, resulting in a catch bond-type behavior (Duke 2000; Caremani et al 2015; Guo and Guilford 2006; Erdmann and Schwarz 2012; Veigel et al 2003; Akalp and Vernerey 2016). Similarly, the head detaches when it is pulled towards rupture of the bond. In our model, the force applied on a myosin head is proportional to the variable $s+y$, so we define two functions of this variable, $\bar{k}_{- \text {pre }}$ and $\bar{k}_{- \text {post }}$ describing the detachment rates in pre- and in post-power stroke, respectively, see Fig.3(c). Finally, the detachment rate is given by

$$
\begin{aligned}
k_{-}(s, y)=\psi_{1_{\text {pre }}}(y) \bar{k}_{- \text {pre }}( & +y) \\
& +\psi_{1_{\text {post }}}(y) \bar{k}_{- \text {post }}(s+y) .
\end{aligned}
$$

The attachment and detachment rates $k_{+}$and $k_{-}$ are visualized in the $(s, y)$ plane in Figs. 4(a)-(b), together with the contour lines of the energy landscape in the detached and attached states, respectively. When the fiber shortens, the value of $s$ decreases and eventually enters the interval around $s=0$ where the attachment rate is highest, and this also corresponds to the region of the relaxed configuration for the pre-power stroke detached head, see label (1) in Fig. 4(a). When the cross-bridge is attached, force is produced as long as the cross-bridge is in tension, i.e. $s+y>0$.

During shortening, the system is driven by the gradient of the energy landscape - in the $y$ direction - towards the post-power stroke state, see (2) $\rightarrow$ (3) in Fig. 4(b). When $s<-a$ (here $a=11 \mathrm{~nm}$ ), the gradient of the energy landscape is such that the cross-bridge becomes compressed and therefore counterproductive, hence the rapidly increasing detachment rate. Similarly when the muscle is lengthened, the cross-bridge should rapidly detach to allow the force to relax.

In Fig. 4(c) we represent the rates introduced in (36), which integrate all possibilities of attachment and detachment, i.e. from all possible values of the power stroke variable $y$, assumed to be distributed according to its equilibrium probability density at fixed $s$, namely $p_{1}^{t h}$, see (34).

5.2 Parameter calibration based on the thermal equilibrium model

In this section, we will calibrate all the remaining parameters - except for the drag coefficient $\eta$ that will be dealt with in Section 5.3.2 - based on the thermal equilibrium model and manipulations thereof to determine the effect of the parameters, and using experimental data for isometric contraction, quick recovery of force in fast isometric transients, and force-velocity relation.

\subsubsection{Isometric contraction}

One of the most usual experiments conducted on a single muscle fiber is the measurement of isometric tension, which is the maximum active force that the fiber can generate under fixed length condition (McMahon 1984). The sarcomere length directly affects the degree of overlap of the myofilaments, and therefore controls the number of cross-bridges that are available for attachment. This phenomenon is at the origin of the so-called Frank-Starling effect in cardiology (Moss and Fitzsimons 2002; Niederer and Smith 2009; Caruel et al 2014). However, in this paper we do not consider this effect, and instead assume that the experiments are performed in the region of optimal overlap between the my- 

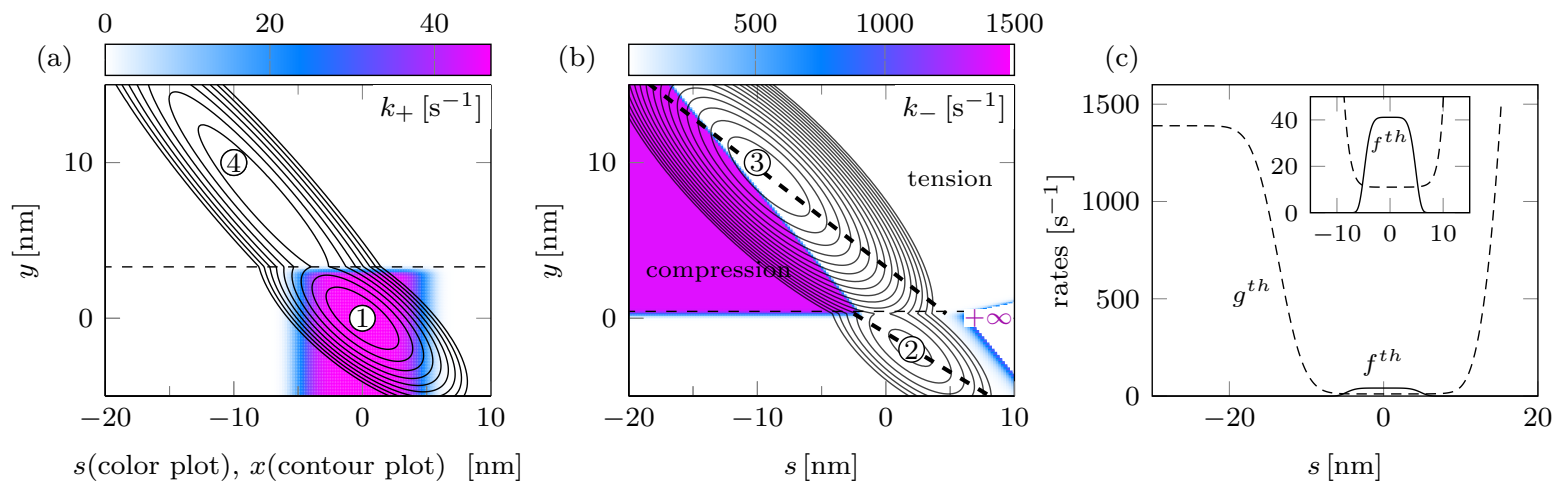

Fig. 4 (a) and (b): Attachment $k_{+}(s, y)$ and detachment $k_{-}(s, y)$ rate functions; the color map represents the attachment and detachment rates, and the black contours show the energy levels in the detached (a) and attached (b) states; the thick dashed line in (b) indicates the locus of the minimum of the energy at fixed $s$. (c) Reaction rates obtained from (36), corresponding to the integral of the contours in (a) and (b) with respect to the equilibrium densities (34).

ofilaments, which means that all cross-bridges can be recruited for attachment. The typical isometric stress measured on frog sartorius muscle (rana esculenta), under physiological conditions, is of the order of $160 \mathrm{kPa}$ (Brunello et al 2014). In such conditions about $22 \%$ of the cross-bridges are attached to actin filaments. In the steady state regime under isometric conditions, we may reasonably assume that all the internal degrees of freedom of the cross-bridges are distributed according to their thermal equilibrium probability density, see (34). Hence, denoting by $\check{P}_{1}^{t h}(s)$ the probability for a head - in thermal equilibrium and isometric condition - located at position $s$ to be attached, (19) leads to

$\check{P}_{1}^{t h}(s)=\frac{f^{t h}(s)}{f^{t h}(s)+g^{t h}(s)}$,

where $f^{\text {th }}$ and $g^{\text {th }}$ are the equilibrium attachment and detachment rates given by (36), respectively. Then the fraction of attached myosin heads can be computed as follows

$\check{n}_{1}^{t h}=\frac{1}{d_{a}} \int_{s=s_{-}}^{s_{-}+d_{a}} \check{P}_{1}^{t h}(s) d s$.

Similarly, the average isometric tension generated by a myosin head (available for attachment) is given by

$\check{\tau}_{c}^{t h}=\frac{1}{d_{a}} \int_{s=s_{-}}^{s_{-}+d_{a}} \check{P}_{1}^{t h}(s) \tau_{c}^{t h}(s) d s$,

where $\tau_{c}^{t h}(s)$ is the average tension generated by crossbridges attached at $s$, i.e.

$\tau_{c}^{t h}(s)=\int_{y} \kappa(s+y) p_{1}^{t h}(y ; s) d y$, with $p_{1}^{t h}$ given by (34). Note that this quantity is independent of the isometric assumption. The average stiffness per myosin can also be computed as

$\check{\kappa}_{c}^{t h}=\frac{1}{d_{a}} \int_{s=s_{-}}^{s_{-}+d_{a}} \check{P}_{1}^{t h}(s) \partial_{s} \tau_{c}^{t h} d s$.

This corresponds to variations of $\check{\tau}_{c}^{t h}$ per unit length of extension variation, for such variations assumed to be slow enough to preserve thermal equilibrium and fast enough to not allow binding or unbinding.

As in Section 4.1, the above quantities have to be rescaled to obtain the macroscopic isometric tension per unit cross section $T_{0}=\rho_{m} \ell_{h s} \check{\tau}_{c}^{t h}$, and the corresponding stiffness quantity $K_{0}=\rho_{m} \ell_{h s}^{2} \check{\kappa}_{c}^{t h}-$ using here the standard muscle physiology notation $\left(T_{0}, K_{0}\right)$ for isometric contraction. We point out that $\rho_{m} \ell_{h s}=\rho_{\text {sarc }} N$, where $\rho_{\text {sarc }}$ denotes the density of myosin filaments in the muscle cross section, and $N$ the maximum number of cross-bridges per myosin filament, and therefore

$T_{0}=\rho_{\text {sarc }} N \check{\tau}_{c}^{t h}, \quad K_{0}=\rho_{\text {sarc }} N \ell_{h s} \check{\kappa}_{c}^{t h}$,

for which typical values of the scaling coefficients are $N=300$, and $\rho_{\text {sarc }}=5 \times 10^{14} \mathrm{~m}^{-2}$, see (Mobley and Eisenberg 1975; Reconditi 2006).

The isometric distribution $\check{P}_{1}^{\text {th }}$ is shown in Fig. 5(a), together with the rates $f^{t h}$ and $g^{t h}$. With the chosen attachment and detachment rate functions, the distribution is rather uniform on the interval where the attachment function is positive (dotted line). Hence, the fraction of cross-bridges attached in isometric contraction can be inferred from the ratio of the width of the distribution over the distance between two binding sites, and the ratio between the plateau values of $f^{t h}$ and $g^{t h}$. The value of the maximum attachment rate $k_{1}$ is adjusted so that the maximum of $f^{t h}$ is of about $40 \mathrm{~s}^{-1}$, in accordance with (Caremani et al 2015). The minimum 

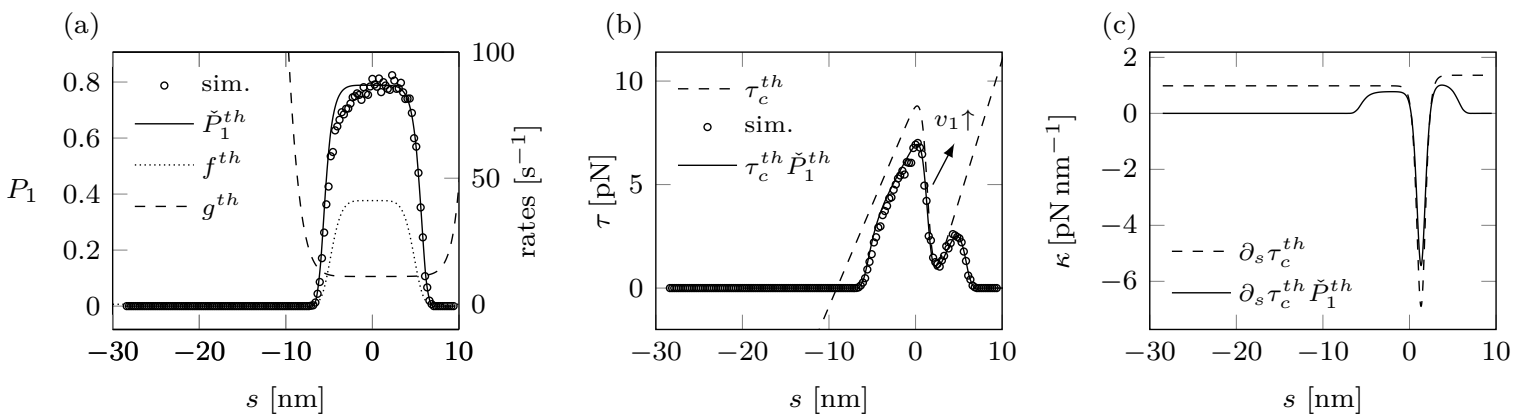

Fig. 5 Isometric contraction. (a) Steady state probability of attachment $\check{P}_{1}^{t h}$ (solid) shown with the equilibrium attachment (dotted line) and detachment (dashed line) rates. [(b) and (c)] Dashed lines, thermal equilibrium tension (b) and stiffness (c) of an attached myosin head; solid lines represent distribution of force (b) and stiffness (c). Circles in (a)-(b) represent average values obtained with stochastic simulations.

value of the detachment rate $k_{\min }$ is also chosen based on (Caremani et al 2015) where we picked a value corresponding to the detachment from an intermediate stage of the power stroke, namely $k_{\min }=11 \mathrm{~s}^{-1}$. The supports of the rate functions $\bar{k}_{+}, \bar{k}_{- \text {pre }}$ and $\bar{k}_{- \text {post }}\left(\lambda_{1,2,3}\right.$ and $\left.s_{ \pm}\right)$ are then adjusted so as to provide a width of the distribution $\check{P}_{1}^{t h}$ leading to a fraction of attached heads $\check{n}_{1}^{\text {th }}$ - see Table 2 - consistent with values found in the literature, such as in (Piazzesi et al 2007; Brunello et al 2014; Linari et al 1998).

Once the distribution $\check{P}_{1}^{t h}$ is calibrated, the parameters of the bistable potential $u_{1}$ can be adjusted so that the equilibrium isometric tension coincides with experimental values which are of the order of $5-6 \mathrm{pN}$ per attached head (Brunello et al 2014). The energy of an attached cross-bridge depends on five parameters (see Tab. 1$)$, namely $a, \kappa_{1 \text { pre }}, \kappa_{1 \text { post }}, \ell_{1}$ and $\kappa$. The power stroke characteristic distance $a=11 \mathrm{~nm}$ has already been set based on literature data as discussed above. Moreover, several experimental techniques also allow an indirect measurement of the stiffness parameter $\kappa$, which is taken here as $\kappa=2.72 \mathrm{pN} . \mathrm{nm}^{-1}$, see (Linari et al 1998; Piazzesi et al 2007; Linari et al 2007; Fusi et al 2014; Linari et al 2009; Brunello et al 2014). The stiffness parameters of the pre- and post-power stroke states $\left(\kappa_{1_{\text {pre }}}\right.$ and $\left.\kappa_{1_{\text {post }}}\right)$ are directly constrained to reproduce the fast isometric transient experiments (see Section 5.2.2), so the only remaining parameter is $\ell_{1}$, i.e. the position of the energy barrier on which depends the intrinsic energy bias of the attached potential, $v_{1}$. The effect of increasing $v_{1}$ is illustrated by the arrow in Fig. 5(b). When $v_{1}$ increases $-\ell_{1}$ decreases - the isometric force per head increases as the equilibrium density moves towards the post-power stroke state. A direct adjustment of the position of the energy barrier allows to find $\check{\tau}_{c}^{t h}=1.14 \mathrm{pN}$, which is the average force per head including the detached ones, and this corresponds to an average force per attached head of $5.1 \mathrm{pN}$, in ac- cordance with experimental data reported in (Brunello et al 2014). After rescaling, we obtain the macroscopic value $T_{0}=171 \mathrm{kPa}$, which is physiologically relevant.

Similarly, the obtained average stiffness per head is $\check{\kappa}_{c}^{t h}=22 \times 10^{-3} \mathrm{pNnm}^{-1}$. This corresponds to a stiffness per attached head of $0.1 \mathrm{pNnm}^{-1}$ and to a macroscopic modulus $K_{0}=3.27 \mathrm{MPa}$. Notice that the above stiffness quantity $\check{\kappa}_{c}^{\text {th }}$ is different from the stiffness $\kappa$ of the myosin head. The latter can be interpreted as an instantaneous stiffness that can probed using for instance rapid out of equilibrium shortening or lengthening of a muscle fiber, see Section 5.2.2. The former is defined in (47) and can be interpreted as a stiffness associated with a slower timescale - typically $1 \mathrm{~ms}$ - see also Section 5.2.2 for greater detail. The calibration results concerning the isometric contraction are summarized in Table 2.

At this stage of the calibration, the only parameters remaining to be adjusted are those of the detached potential $u_{0}$. To our best knowledge, there is no direct characterization for the mechanical properties of the detached heads, which gives a large leeway for the parameters of the $u_{0}$ energy landscape. However, the shape of the double well potential dictates the timescale for the repriming of the power stroke mechanism, which is an important element in the calibration the model. In our case, we adjust the detached energy function $u_{0}$ so that the Kramers theory estimate of the equilibration timescale is about $7 \mathrm{~ms}$.

\subsubsection{Fast isometric transients}

The remaining parameters of the cross-bridge energy in the attached state are the stiffnesses $\kappa_{1 \text { pre }}$ and $\kappa_{1_{\text {post }}}$. These two parameters can be inferred from mechanical experiments focussing on the so-called quick force recovery during fast isometric transients. The protocol was originally designed by A.F. Huxley and R.M. Sim- 
Table 2 Main quantities characterizing the state of isometric contraction.

\begin{tabular}{|c|c|c|c|c|}
\hline \multirow[t]{2}{*}{ Parameter } & \multirow[t]{2}{*}{ symbol } & \multicolumn{2}{|c|}{ values } & \multirow[t]{2}{*}{ unit } \\
\hline & & Thermal eq. & Stochastic & \\
\hline Fraction of cross-bridge attached & $\check{n}_{1}$ & 0.222 & 0.217 & \\
\hline Number of attached heads / half-sarc. & $\check{N}_{1}$ & 67 & 65 & \\
\hline Total stress & $T_{0}$ & 171 & 165 & $\mathrm{kPa}$ \\
\hline Tension per head & $\check{\tau}_{c}$ & 1.14 & 1.1 & $\mathrm{pN}$ \\
\hline Tension per attached head & $\check{\tau}_{c 1}$ & 5.12 & 5.07 & $\mathrm{pN}$ \\
\hline Average stiffness per head $(\neq \kappa)$ & $\check{\kappa}_{c}$ & $22 \times 10^{-3}$ & $18 \times 10^{-3}$ & $\mathrm{pN} \mathrm{nm}^{-1}$ \\
\hline Average stiffness per attached head & $\check{\kappa}_{c 1}$ & 0.1 & 0.08 & $\mathrm{pN} \mathrm{nm}^{-1}$ \\
\hline Active modulus & $K_{0}$ & 3.27 & 2.73 & $\mathrm{MPa}$ \\
\hline
\end{tabular}

mons in (Huxley and Simmons 1971), then extensively used and improved ever since, see e.g. (Ford et al 1977; Lombardi et al 1992; Piazzesi et al 2002). An isometrically contracting muscle fiber is submitted to rapid ( 100 us) length changes while the evolution of the tension is recorded. The mechanical response of the fibers to shortening steps of various sizes was shown to comprise four phases. Phase 1 corresponds to the purely elastic response, which takes place during the applied length step itself. The tension at the end of this phase is denoted by $T_{1}$. During phase 2 , the tension recovers partially its original value within a few milliseconds. The end of phase 2 is signaled by a plateau in the time evolution of the tension. The tension at the plateau is denoted by $T_{2}$. This timescale is known to be too small to allow the process of attachment-detachment to take place. Therefore, phase 2 happens with a constant number of attached motors. The rise in tension is attributed to the power stroke mechanism, which allows the attached cross-bridges to generate force upon performing the conformational change. The quick force recovery observed during phase 2 thus corresponds to the relaxation of the attached cross-bridge towards the thermal equilibrium configuration associated with the new fiber length.

Phases 3 and 4 are happening on a timescale of more than $10 \mathrm{~ms}$. They correspond to the initiation and the steady state regime of the attachment / detachment process, which allows to return to the initial isometric tension corresponding to the new fixed length.

Taking the isometric contraction as the initial equilibrium state, we consider an instantaneous displacement of the actin filament, which we denote by $\delta s$. We assume that phase 1 is sufficiently fast to consider that the distribution inside the double well energy landscape does not change, i.e. for any given attached head, $y$ remains constant. As a result, the variation of the tension is solely due to the change in $x=s$, so that for a head attached at $s$ the tension variation is $\kappa \delta s$. Therefore the tension per head at the end of the step can be written as $\tau_{1}(\delta s)=\check{\tau}_{c}^{t h}+\kappa \check{n}_{1}^{t h} \delta s$, with $\check{\tau}_{c}^{t h}$ the isometric force per head given in (45), and $\check{n}_{1}^{\text {th }}$ the fraction of attached head in isometric condition in (44). After rescaling, this gives the total stress at the end of phase 1

$T_{1}(\delta s)=T_{0}+\rho_{\text {sarc }} N \kappa \check{n}_{1}^{t h} \delta s$.

At the end of phase 2, the power stroke variable $y$ of all attached heads is distributed according to its equilibrium density at the new position of the binding site. Hence, the tension $T_{2}$ attained at the end of phase 2 can be computed from

$T_{2}(\delta s)=\rho_{\text {sarc }} N \frac{1}{d_{a}} \int_{s=s_{-}}^{s_{-}+d_{a}} \check{P}_{1}^{t h}(s) \tau_{c}^{t h}(s+\delta s) d s$,

where $\check{P}_{1}^{t h}$ is the isometric density of attached head (43) and $\tau_{c}^{t h}$ is the thermal equilibrium tension of an attached cross-bridge (46). Note that the slope of the $T_{2}(\delta s)$ curve around $\delta s=0$ gives a stiffness quantity that is directly associated with the individual myosin stiffness computed in (47).

The above computation cannot be used directly to confront our results with the experimental data. Indeed, the experimental apparatus controls the length step at the level of the half sarcomere, and not directly of the myofilaments. To compare our results with the experimental data, we must take into account the compliance of the filaments, which we represent as a linear elastic element in series with the cross-bridge array. We denote by $C_{m}$ the compliance of the myofilament, taken from (Brunello et al 2014) as $C_{m}=13 \mathrm{~nm} \mathrm{MPa}^{-1}$. Then, at the end of phase 1 and phase 2 , we can deduce the total length change per half sarcomere $\delta \ell_{h s}$ from our displacements $\delta s$ by adding the corresponding filament length change. The total length changes are thus given by

$$
\begin{aligned}
\delta \ell_{h s}(\delta s) & =\delta s+C_{m}\left[T_{1}(\delta s)-T_{0}\right], \\
\delta \ell_{h s}(\delta s) & =\delta s+C_{m}\left[T_{2}(\delta s)-T_{0}\right],
\end{aligned}
$$

for phase 1 and phase 2 , respectively. We can then check in Fig. 6(b) that the $T_{1}$ curve that we obtained is in adequacy with experimental data, as expected since the 
stiffness parameter $\kappa$ was taken from experimentally calibrated values.

For the calibration of the $T_{2}$ curve, in order to more clearly identify the associated parameter sensitivities, we observe that for large shortening steps the tension $\tau_{c}^{t h}(s)$ becomes a linear function of $s$, due to the fact that almost all the attached cross-bridges are in the post-power stroke state and thus have the same stiffness $\kappa_{1 \text { post }}$, see Fig. 6(a). We can then use the approximation $\tau_{c}^{t h}(s+\delta s) \approx \frac{\kappa_{1 \text { post }} \kappa}{\kappa+\kappa_{1 \text { post }}}\left(s+\delta s+a-s_{1}\right)$ in (50). With the chosen rate functions, we can also consider that the distribution of the attached state $\check{P}_{1}^{t h}$ is constant over an interval $\left[s_{-}^{i s o}, s_{+}^{i s o}\right]$ centered around $s=0$. With these two approximations, (50) simplifies into

$T_{2}\left(\delta_{s}\right) \approx \rho_{\text {sarc }} N \check{n}_{1}^{t h} \frac{\kappa_{1_{\text {post }}} \kappa}{\kappa+\kappa_{1_{\mathrm{post}}}}\left(\delta s+a-s_{1}\right)$,

which allows to calibrate the parameters $\kappa_{1 \text { post }}$ and $s_{1}$.

The results of our calibrations are shown in Fig. 6. In (a) we show how $T_{2}$ is computed, as an illustration of (50). In (b) the results of these computations (dashed line for phase 1 and solid line for phase 2) are compared to experimental data (rectangles for phase 1 and circles for phase 2) gathered from (Ford et al 1977; Brunello et al 2007; Linari et al 2009).

The $T_{2}$ curve shows a plateau near the isometric point at $\delta \ell_{h s}=0$ - see Fig. $6(\mathrm{~b})-$ which is the consequence of the relatively low value of the modulus $\check{\kappa}_{c}^{t h}$, since the slope of $T_{2}$ at $s=0$ is directly given by $\rho_{\text {sarc }} N \check{\kappa}_{c}^{t h}$.

The transient response to fast length changes in stretching was much less investigated experimentally than in shortening. In (Piazzesi et al 1997) and (Brunello et al 2007), such experiments were conducted, but the presence of a linear part of the $T_{2}$ curve in stretching is much less clear than in shortening. Without further information about the behavior in stretching, our parameter $\kappa_{1 \text { pre }}$ remains poorly constrained. The value $\kappa_{1_{\text {pre }}}=2.72 \mathrm{pN} \mathrm{nm}^{-1}$ was chosen arbitrarily but close to the value of the post-power stroke stiffness, $\kappa_{1_{\text {post }}}=$ $1.57 \mathrm{pN} \mathrm{nm}^{-1}$, see Table 1 and Fig. 5(c) (dashed line).

\subsubsection{Force-velocity relation}

Another major feature for characterizing the mechanical behavior of a muscle is the so-called force-velocity relation, i.e. the maximum shortening speed under a given external force. Here, we follow a slightly different protocol and prescribe a constant shortening velocity $\dot{s}=\dot{x}_{c}$. One can derive an approximation of the force-velocity relation by again assuming that the power stroke element equilibrates faster than the characteristic time of the attachment-detachment process and consider the dynamics of the two state dynamic equation
(19), with the rates (36). In the steady state regime, we have

$\dot{x}_{c} \partial_{s} P_{1}=f^{t h}(s)\left[1-P_{1}\right]-g^{t h}(s) P_{1}$,

which can be solved numerically for different values of $\dot{x}_{c}$. Energy landscapes and the attachment rate have been calibrated in Sections 5.2.1 and 5.2.2. Here we adjust the detachment function using the force-velocity relation measured in (Edman and Hwang 1977).

Numerical solutions of (53) and the corresponding force distributions are represented in Figs. 7(a)-(b) for various shortening speeds. The dotted lines represent the distributions in isometric conditions, already shown in Fig. 5. As the shortening velocity increases, the distribution spreads towards negative values of $s$. As a result, some attached cross-bridges are drifted in a region where the equilibrium force becomes negative, see Fig. 7(b). When the population of compressed elements equilibrates the population of cross-bridges in tension, the total average force is equal to 0 . The corresponding velocity is the maximum shortening velocity - often denoted by $V_{\max }$ - that the muscle fiber can sustain. The value of $V_{\max }$ is controlled by the detachment function $\bar{k}_{- \text {post }}$, which is essentially characterized by the threshold position $\ell_{-}$and the amplitude $k_{0}$. Decreasing the value of $\ell_{-}$and the value of $k_{0}$ will increase $V_{\max }$, since the point where the compressed cross-bridges compensate those in tension will correspond to a higher shortening velocity. We have chosen the value of $k_{0}=1378 \mathrm{~s}^{-1}$, consistent with the value reported in (Caremani et al 2015) (parameter $k_{5}$ ), and $\ell_{-}$was then used as the sole adjustment parameter to obtain an adequate value of $V_{\max }$ for the full stochastic model, which provides a value larger than the experimental characterization of (Edman and Hwang 1977) with the thermal equilibrium model, see Fig. 7(c).

\subsection{Results obtained using the full stochastic model}

In the previous section, the calibration was mostly performed using the simplified model by which the internal degree of freedom $y$ representing the orientation of the myosin head was assumed to be in thermal equilibrium. We will now report on detailed Monte-Carlo simulations of the complete model (typically with 10000 particles in each simulation and a time step of $0.4 \mu \mathrm{s}$ ).

\subsubsection{Typical trajectories}

To illustrate the above calibration of the energy landscapes and the rates, we present a typical trajectory of a single motor submitted to an imposed sliding of 


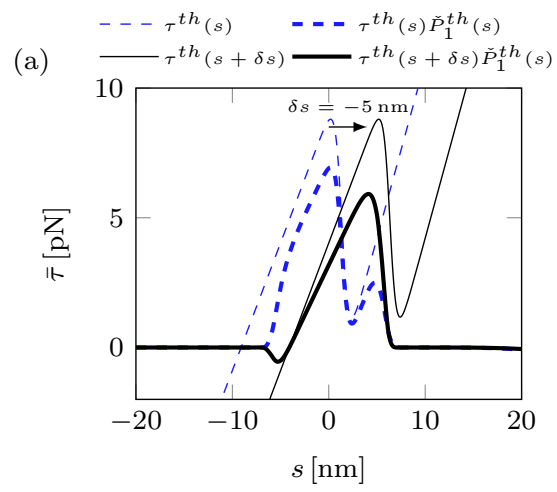

(b)

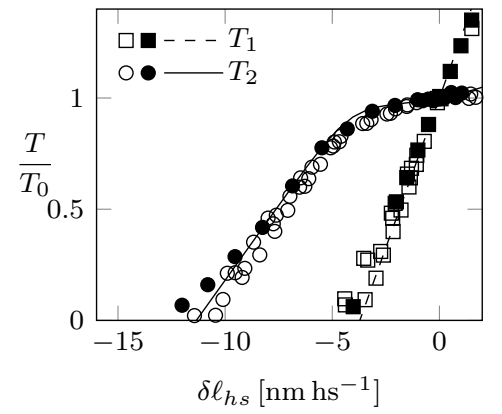

(c)

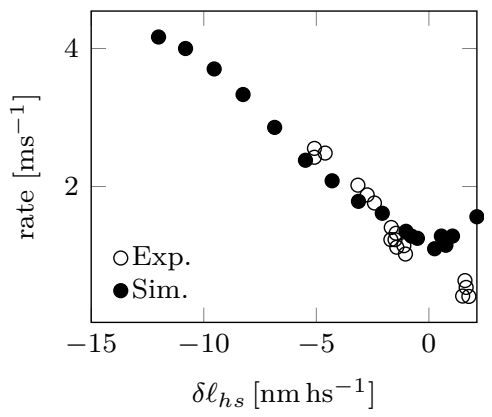

Fig. 6 Calibration with the quick recovery experiments. (a) Effect of a shortening of $4 \mathrm{~nm} \cdot \mathrm{hs}^{-1}$ on the distribution of force. Thin lines, thermal equilibrium tension $\tau_{c}^{t h}$ [see (46)] before (dashed, blue), and after the step (solid, black). Thick lines, distribution of tension $\tau_{c}^{t h}(s) \check{P}_{1}^{t h}(s)$ [see (50)], before (dashed, blue), and after the step (solid, black). (b) Comparison with experimental data (open symbols, from (Ford et al 1977; Brunello et al 2007; Linari et al 2009)) and with the direct stochastic simulations (filled symbols); dashed line and squares for $T_{1} / T_{0}$; solid line and circles for $T_{2} / T_{0}$. (c) Rate of force recovery obtained from the stochastic simulations (filled symbols) compared with experimental data (open symbols from (Linari et al 2009; Ford et al 1981; Lombardi et al 1992)).

(a)

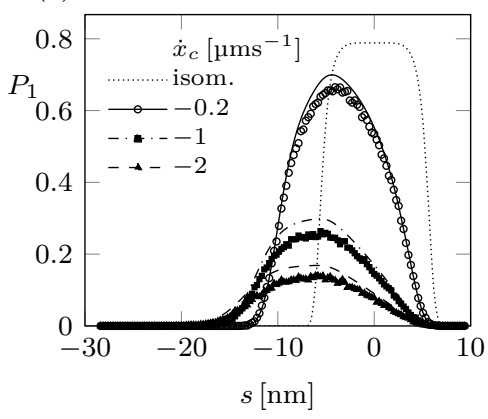

(b)

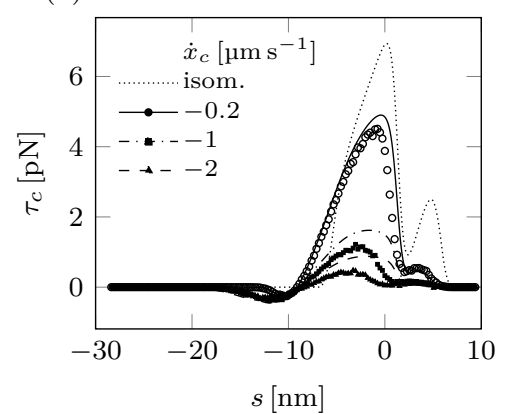

(c)

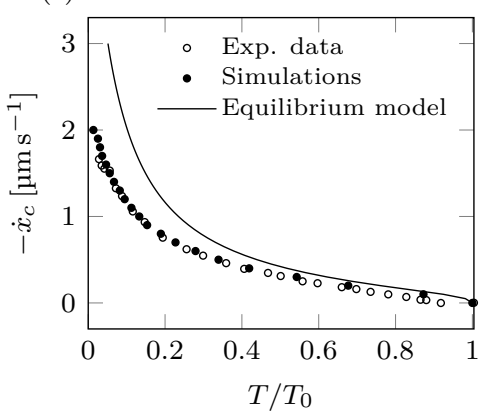

Fig. 7 Response of the model to a prescribed shortening velocity. [(a) and (b)] steady state distribution of attached crossbridges (a) and force (b); dotted and solid lines, results obtained using (53); symbols, distributions obtained with direct stochastic simulations (Monte-Carlo). (c) Force-velocity relation obtained from (Edman and Hwang 1977) (open symbols), from the model (53) (solid line) and from direct stochastic simulations (filled symbols).

the filament at constant shortening rate, see Fig. 8. The four steps of the Lymn-Taylor cycle are well reproduced. When the head binds - see (2) in Fig. 8(a) - it executes the power stroke before detaching when the tension becomes negative - see (3) and (4).

\subsubsection{Fast isometric transients and associated timescales}

We have performed complete stochastic simulations of the isometric transients already discussed in Section 5.2.2. An example of the response to a rapid shortening of $4 \mathrm{~nm}$ is shown in Fig. 9. We checked that within the time of the quick force recovery, the fraction of attached heads changes by less than $1 \%$, which supports the theory according to which the force recovery is due to the relaxation of the power stroke mechanism. We computed the simulated tension levels $T_{1}$ and $T_{2}$ associated with varying values of length variations following the method proposed in (Ford et al 1977) - see Fig. 9 - and the results are shown in Fig. 6(b), compared with experimental data found in (Piazzesi et al 1992; Linari et al 2009; Ford et al 1977). We note that the predicted values are in excellent agreement with those obtained with the thermal equilibrium model and with the experimental values.

In addition, the fast isometric transient provides a direct way to calibrate the timescale of the stochastic dynamics. The so-called quick force recovery - second phase of the fast isometric transient response - corresponds to the relaxation of the internal degree of freedom $y$ towards a new equilibrium. The dynamics of this process is supposedly independent of the attachmentdetachment kinetics, and is therefore determined by the value of the microscopic timescale $\gamma=\eta / \kappa$, where $\eta$ is an unknown drag coefficient - that remains to be calibrated in our case - and $\kappa$ is the stiffness of the crossbridge, see Table 1 . We consider the rate of of phase 


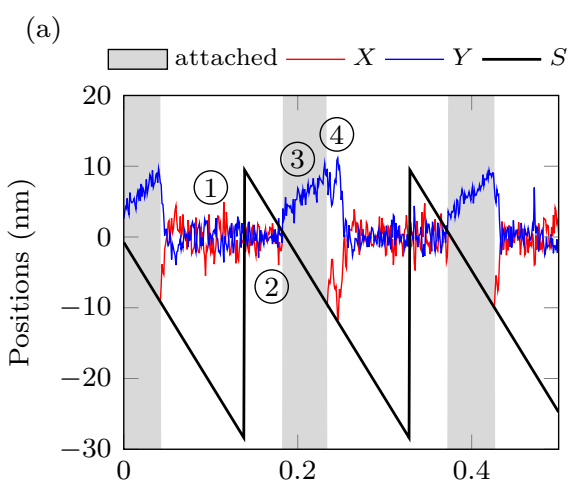

(c)
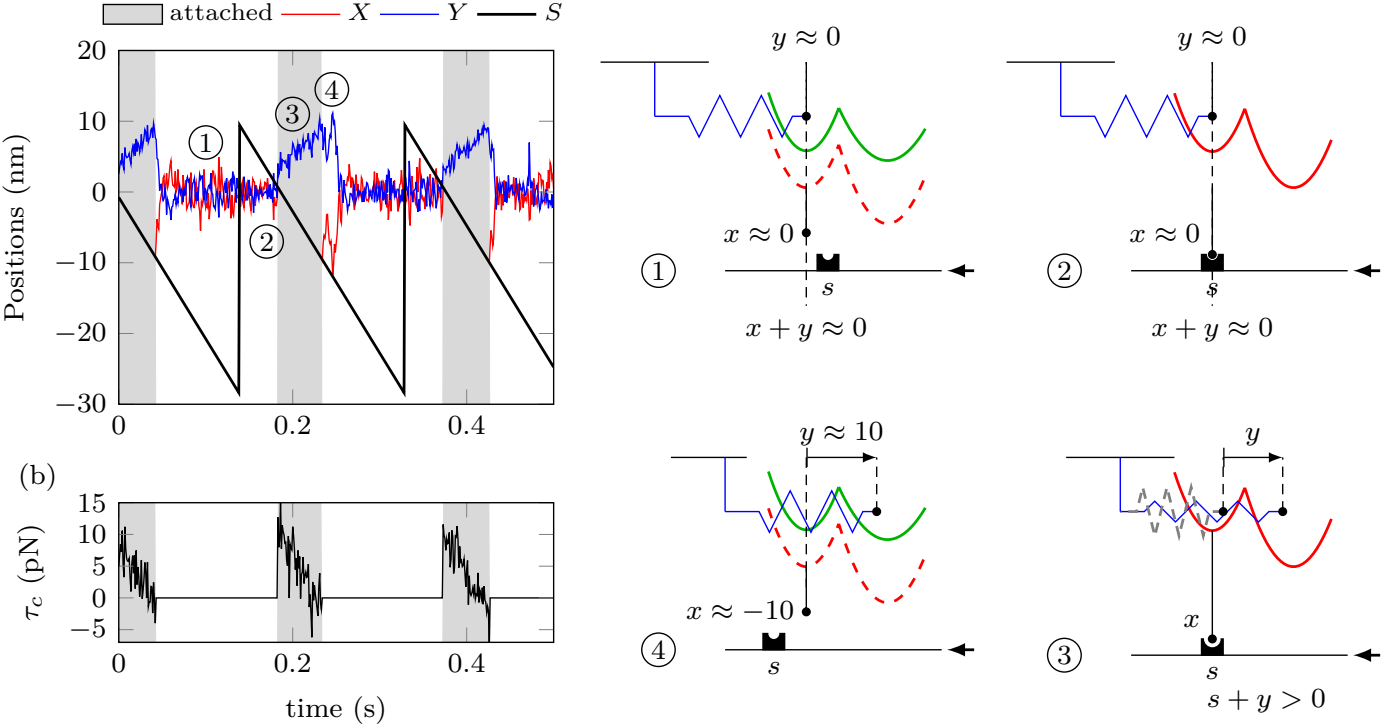

Fig. 8 Behavior of a single myosin head under steady-state shortening $\dot{s}=-0.2 \mu \mathrm{ms}^{-1}$. (a) Time evolution of $X$ (red), $Y$ (blue) and $s$ (black), with gray bands when the cross-bridge is attached. (b) Corresponding evolution of the tension. (c) Typical four steps of the Lymn-Taylor cycle: (1) detached cross-bridge in pre-power stroke; (2) attachment when a binding site comes near the head; (3) post-power stroke configuration; (4) detachment when cross-bridge starts being compressed.

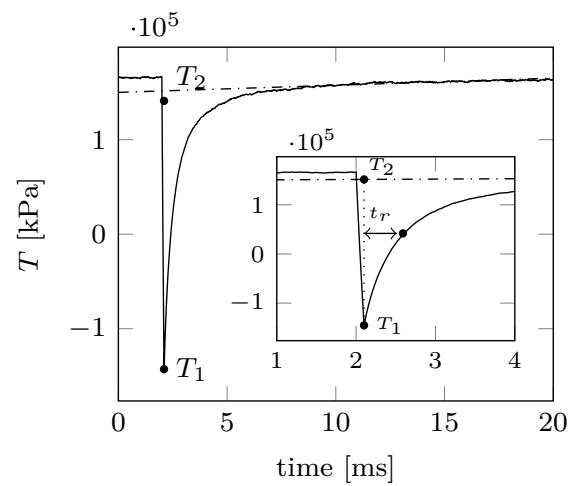

Fig. 9 Tension recovery after a shortening step of $\delta s=$ $-4 \mathrm{~nm}$ applied at time $t=2 \mathrm{~ms}$. The tension $T_{1}$ is identified as the tension at the end of the shortening step. The tension $T_{2}$ is obtained following the procedure described in (Ford et al 1977), see dash-dotted line. The rate of tension recovery shown in Fig. 6(c) corresponds to $1 / t_{r}$, where $t_{r}$ represent the time to reach the tension $T_{1}+0.63\left(T_{2}-T_{1}\right)$ starting from $T_{1}$, see inset with zoomed timescale.

2 - as measured experimentally - as the inverse of the time $t_{r}$ taken by the fiber, starting from the end of phase 1 at tension $T_{1}$ to reach $T_{1}+0.63\left(T_{2}-T_{1}\right)$, which corresponds to an estimate of the characteristic time of an exponential decay, see Fig. 9. By adjusting only $\eta$ - the last remaining parameter to be calibrated - our model is able to fit the timescale of the quick force recovery, see Fig. 6(c), where we do not provide results for steps smaller than $1 \mathrm{~nm} \mathrm{hs}^{-1}$ due to uncertainties related to noise level.

\subsubsection{Isometric contraction}

We simulated isometric contraction by setting the sliding velocity to zero in the stochastic equations, initializing the system with all myosin heads in the detached state and with randomly chosen values of $X$ and $Y$. The corresponding mean fraction of attached cross-bridges and the associated mean tension obtained from the statistics of the stochastic trajectories - after running the simulation for a time sufficient to reach steady-state - are shown in Fig. 5(a)-(b). A direct mean over $s$ then gives the values of the overall fraction of attached bridges and average tension reported in Table 2 . The stiffness values provided in the same column of this table are defined and computed as the slopes of the $T_{2}$-tension curve at the origin $(\delta s=0)$ for the above force recovery experiments. The results obtained with the full stochastic model are consistent with the values obtained with the thermal equilibrium model, which confirms that the latter can be used as an effective calibration tool.

\subsubsection{Force-velocity relation}

The force-velocity curve can also be obtained from direct stochastic simulations. To that purpose, the system is first maintained in isometric condition until it reaches a steady state, and then a ramp shortening is applied with a prescribed velocity. The results are shown - and 
compared with those of the thermal equilibrium model - in Fig. 7(c).

\section{Discussion}

Direct and inverse chemical reactions From a fundamental point of view, the reaction rates $k_{+}$and $k_{-}$in fact each aggregate one direct and one inverse reaction rate, as the pre-power-stroke attachment reaction is itself associated with an inverse detachment reaction, and likewise the post-power-stroke detachment reaction consuming one ATP has an inverse attachment reaction, see e.g. Hill (2004). As the direct and inverse rates of any single reaction are related to the free energies via the so-called detailed balance equation

$\frac{k_{A \rightarrow B}}{k_{B \rightarrow A}}=\exp \left[-\frac{w_{B}-w_{A}}{k_{B} T}\right]$

this implies that - strictly speaking - once the free energies have been adjusted, one should choose the direct reaction rates for each reaction, and then the inverse rates would be directly inferred, hence also $k_{+}$and $k_{-}$. However, recalling Fig. 2(a) our energy levels are adjusted in such a way that each direct reaction is associated with a significant drop in energy, and therefore we have implicitly resorted to the approximation of neglecting the reverse reaction rates and directly calibrated the aggregated rates $k_{+}$and $k_{-}$. Note that a factor 100 for the ratio of direct to inverse reaction rates corresponds to a drop in energy of about $20 \mathrm{zJ}$ at experimental temperatures, indeed.

Detached energy landscape In Section 5.2.1, we calibrated the detached energy landscape so that the equilibration timescale is about $15 \mathrm{~ms}$. This value is somewhat shorter than that chosen in (Caremani et al 2015) - about $40 \mathrm{~ms}$ - but our choice ensures that the equilibrium distribution in the detached state is localized predominantly in the pre-power-stroke configuration, which facilitates the identification of $f^{\text {th }}$ with $k_{+}$. In addition, our calibration is more consistent with the use of the thermal equilibrium model in isometric condition, since this requires that the equilibrium in the detached state is reached significantly faster than attachment occurs. Nevertheless, these calibrations can - and should, indeed - be revisited if additional experimental evidence becomes available.

Force recovery rate Note that in Fig. 6(c) the dependence of the recovery rate on the length step is not exponential as incorrectly predicted by the Huxley-Simmons model, see (Huxley and Simmons 1971). For small shortening steps, the double well structure is present - as in
Fig. 2(e) - so one can expect an exponential dependence of the rate on the height of the energy barrier, hence also on the length step. However, at larger steps, when there is no barrier - see e.g. Fig. 2(f) - the force recovery process is limited by diffusion, which implies that the overall characteristic rate becomes constant, see also (Offer and Ranatunga 2016; Caruel and Truskinovsky 2017) for a more extended discussion with experimental evidence.

Differences between the stochastic and thermal equilibrium models in isometric contraction The differences between the two models - visible for values of $s \in$ $[-7,-3] \mathrm{nm}$ in Fig. 5 - can be interpreted as follows. For low values of $s$, the attached equilibrium distribution $\bar{p}_{1}^{t h}$ strongly favors the post-power stroke state, see Fig. 2(f), which means that the cross-bridges concerned will undergo the full cycle while the cross-bridges located at higher values of $s$ will remain attached without undergoing the power-stroke, see Fig. 2(d). We have mentioned above that the equilibration timescale in the detached state was not significantly smaller that the characteristic time of attachment, which limits the applicability of the thermal equilibrium hypothesis in the case when the motors have to undergo the full cycle.

Differences between the stochastic and thermal equilibrium models in the force-velocity relation To gain insight as regards the differences observed between the thermal equilibrium and stochastic models in Fig. 7(c), as the thermal equilibrium model does not incorporate - by definition - any viscosity effect, we have run stochastic simulations when varying the value of the viscosity parameter $\eta$. The results are shown in Fig. 10. As expected, we do not see any significant variation in the computed values of $T_{1}$ and $T_{2}$ in Fig. 10(a), whereas the force recovery rate is essentially proportional to the inverse of $\eta$, see Fig. 10(b). In the force-velocity relations of Fig. 10(c), the computed tensions are higher - for large velocities - when viscosity is decreased by a factor two.

Important stiffness considerations Recalling the comments made in Section 4.1 regarding the impact of tangent stiffness on tension variations in steady-state sliding, let us now further discuss this effect based on the thermal equilibrium model. We first rewrite (53) as

$$
\dot{x}_{c} \partial_{s} P_{1}=\left(f^{t h}(s)+g^{t h}(s)\right)\left(\check{P}_{1}^{t h}-P_{1}\right) .
$$


(a)

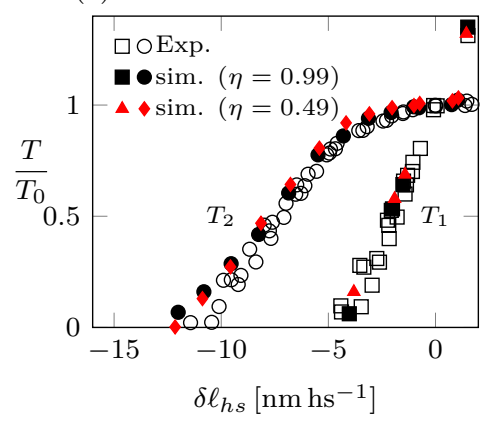

(b)

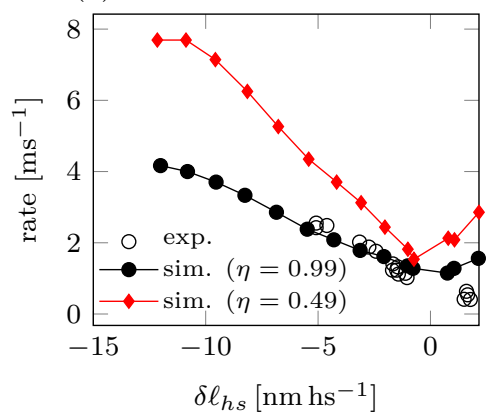

(c)

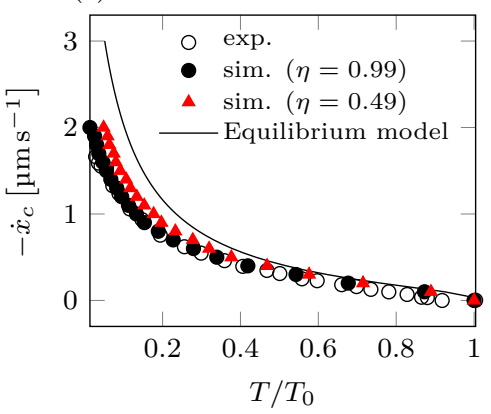

Fig. 10 Effect of a change of the viscosity coefficient $\eta$ on the fast timescale response [(a) and (b)] and on the force-velocity relation (c).

We then have for the active tension

$$
\begin{aligned}
T_{c} & =\rho_{\text {sarc }} N \frac{1}{d_{a}} \int_{s=s_{-}}^{s_{-}+d_{a}} P_{1}(s) \tau_{c}^{t h}(s) d s \\
& =T_{0}-\rho_{\text {sarc }} N \dot{x}_{c} \frac{1}{d_{a}} \int_{s=s_{-}}^{s_{-}+d_{a}} \frac{\partial_{s} P_{1}}{f^{t h}+g^{t h}} \tau_{c}^{t h} d s \\
& =T_{0}+\rho_{\text {sarc }} N \dot{x}_{c} \frac{1}{d_{a}} \int_{s=s_{-}}^{s_{-}+d_{a}} P_{1} \partial_{s}\left(\frac{\tau_{c}^{t h}}{f^{t h}+g^{t h}}\right) d s,
\end{aligned}
$$

for $T_{0}$ given in (48). In order to facilitate the interpretation of the integral term, we will make several approximations. First, we will consider the first-order effect in the sliding velocity $\dot{x}_{c}$, and therefore we can substitute $\check{P}_{1}^{t h}$ for $P_{1}$ in the integral. Then, we can argue that the $s$-differentiation of the denominator $f^{t h}+g^{t h}$ has a limited effect, because variations of $f^{t h}+g^{t h}$ occur when $\check{P}_{1}^{t h}$ is small, and this can be checked numerically, indeed. Moreover, we can consider that $f^{t h}+g^{t h}$ is almost constant - denoted as $\langle f+g\rangle-$ when $\check{P}_{1}^{t h}$ is significant. These approximations yield

$T_{c} \approx T_{0}+\rho_{\text {sarc }} N \frac{\dot{x}_{c}}{<f+g\rangle} \check{\kappa}_{c}^{t h}=T_{0}+\frac{K_{0}}{<f+g\rangle} \dot{e}_{c}$,

where we note the direct similarity with (42) in the translation of the competing effects of tangent stiffness and reaction rates. We now see that there are in essence three major stiffness moduli at play in the system:

- one associated with the slope of the $T_{1}$-curve;

- two associated with the $T_{2}$-curve, i.e. the slope at the origin - which directly conditions the active tension variations in sliding as explained above - and the slope at larger extensions.

Our proposed (complete) model is able to account for all these stiffness quantities, whereas a model of Huxley'57 type can only account for one stiffness, that must then be calibrated to the slope of the $T_{2}$-curve at the origin in order to obtain adequate tension in sliding. As for models of Huxley-Simmons'71 type, they can only reproduce two stiffness moduli, and in particular they give the same slope for the $T_{1}$-curve and for the $T_{2}$-curve at large extensions. Note in passing that the thermal equilibrium reproduces the $T_{2}$-curve very nicely, but of course is not designed to predict the immediate response.

\section{Concluding remarks}

We have proposed a hybrid chemical-mechanical model of myosin heads in sarcomeres, within the classical description of rigid sliding filaments. In our case, myosin heads have two mechanical dofs - one of which associated with the power stroke - and two possible chemical states, i.e. bound in a cross-bridge to an actin site or not. This model is formalized in stochastic equations of Langevin type for each degree of freedom, with jumps associated with attachment-detachment reactions. At the mesoscale level of a population of myosin heads sharing the same filament sliding, we were then able to obtain the corresponding Fokker-Planck partial differential equations governing the probability density functions associated with the mechanical dofs and chemical states. This form is important, as it allows to monitor muscle energetics, in particular. Furthermore, these Fokker-Planck equations are a crucial mediator for comparing our proposed model with classical ones, such as the Huxley'57 model. In fact, starting from this form of our model equations we can infer the Huxley'57 model equations under two different simplifying assumptions: (1) by simply canceling the internal power stroke dof, which brings little physical insight but establishes a Langevin form of the Huxley'57 model, a novel result by itself, and (2) by assuming that evolutions are slow enough for mechanical dofs to constantly remain at thermal equilibrium, which is probably justified in most physiological conditions for striated muscles. In the second case, we thus need to solve the Huxley'57 dynami- 
cal equation pertaining to the ratio of attached heads, but we can then recover the underlying distribution of mechanical dofs - instead of single deterministic extension in Huxley' 57 - by a simple post-processing at each time.

We have then shown how we can calibrate the ingredients of our proposed model - namely, the elastic energies and viscosity parameter for the mechanical dofs and the chemical reaction rate functions - in a systematic manner, based on published experimental data for skeletal muscles. In this calibration process, the thermal equilibrium model has proven to be instrumental, as it provides analytical or numerically cost-free solutions in various conditions. Finally, numerical simulations of our complete model in its Langevin form provided numerical solutions in good to excellent adequacy with all experimental data. In particular, the fast isometric transients - with the force recovery phenomenon - are very well reproduced. In fact, in the above discussion it appeared that our proposed model has the simplest mechanical structure for representing three essential stiffness quantities at play in muscle physiology.

Of course, some leeway remains in the calibration process, in particular in the adjustment of the reaction rates that are complex functions of several variables, and much more extensive assessments relying on more detailed experimental data will be needed to refine this process.

A direct extension of this work concerns similar calibration and assessment of our model based on cardiac - instead of skeletal - data, which may bring some important mechanical insight into differences between the two types of sarcomeres. To that purpose, it should be noted that some invaluable experimental studies have been recently performed (Caremani et al 2016), but some data are still missing or need further confirmation.

Another direct perspective pertains to a more complete assessment of the thermal equilibrium model, and in particular of its detailed applicability - and possible limitations - to represent the physiological behavior of skeletal and cardiac muscles.

Finally, other perspectives are very numerous, but to cite just a few, first of all extending our model to include additional chemical states considered by physiologists is natural and appealing. For example, the so-called off-state of the myosin head could be introduced to investigate possible related mechano-sensing effects, see in particular (Linari et al 2015) and references therein for some experimental evidence on this matter. In the chemical realm as well, we have considered the calcium activation of the thin filaments as given, but of course to complete the coupling with elec- trophysiology some dynamics of calcium activation should be introduced. Likewise, ATP concentration was considered as constant in our model - which is known to be the case in physiological conditions - but some pathological phenomena could be taken into account by modulating this concentration, such as for ischemia.

Acknowledgements: We would like to warmly thank our colleagues from the Laboratory of Physiology of Firenze University - Vincenzo Lombardi and Marco Linari, in particular - for their invaluable feedback on this work, François Kimmig (Ecole Polytechnique and Inria) for insightful discussions on the thermal equilibrium model, and Lev Truskinovsky (ESPCI) for stimulating exchanges on the stochastic model.

Compliance with ethical standards: The authors declare that they have no conflict of interest.

\section{References}

Akalp U, Vernerey FJ (2016) The role of catch-bonds in actomyosin mechanics and cell mechano-sensitivity. Physical Review E 94

Alberts B, Johnson A, Lewis J, Morgan D, Raff M, Roberts K, Walter P (2014) Molecular Biology of the Cell, sixth edn. Garland Science

Bestel J, Clément F, Sorine M (2001) A biomechanical model of muscle contraction. In: Niessen W, Viergever M (eds) Lecture Notes in Computer Science, Springer-Verlag, vol 2208

Brunello E, Reconditi M, Elangovan R, Linari M, Sun Y, Narayanan T, Panine P, Piazzesi G, Irving M, Lombardi V (2007) Skeletal muscle resists stretch by rapid binding of the second motor domain of myosin to actin. PNAS 104(50):20,114-20,119

Brunello E, Caremani M, Melli L, Linari M, FernandezMartinez M, Narayanan T, Irving M, Piazzesi G, Lombardi V, Reconditi M (2014) The contributions of filaments and cross-bridges to sarcomere compliance in skeletal muscle. J Physiol 592(17):3881-3899

Caremani M, Melli L, Dolfi M, Lombardi V, Linari M (2015) Force and number of myosin motors during muscle shortening and the coupling with the release of the ATP hydrolysis products. J Physiol 593(15):3313-3332

Caremani M, Pinzauti F, Reconditi M, Piazzesi G, Stienen GJM, Lombardi V, Linari M (2016) Size and speed of the working stroke of cardiac myosin in situ. PNAS 113(13):3675-3680

Caruel M, Truskinovsky L (2017) Physics of muscle contraction. Rep Prog Phys

Caruel M, Allain JM, Truskinovsky L (2013) Muscle as a metamaterial operating near a critical point. Phys Rev Lett 110

Caruel M, Chabiniok R, Moireau P, Lecarpentier Y, Chapelle D (2014) Dimensional reductions of a cardiac model for effective validation and calibration. Biomechanics and Modeling in Mechanobiology 13(4):897-914, DOI 10.1007/s10237-013-0544-6

Chabiniok R, Moireau P, Lesault PF, Rahmouni A, Deux JF, Chapelle D (2012) Estimation of tissue contractility from cardiac cine-MRI using a biomechanical heart 
model. Biomechanics and Modeling in Mechanobiology 11(5):609-630, DOI 10.1007/s10237-011-0337-8

Chapelle D, Le Tallec P, Moireau P, Sorine M (2012) Energypreserving muscle tissue model: Formulation and compatible discretizations. Journal for Multiscale Computational Engineering 10(2):189-211

Chen YD, Hill TL (1974) Analysis of a Simple Prototypal Muscle Model Near to and Far from Equilibrium. Proc Natl Acad Sci USA 71(5):1982-1986

Dominguez R, Freyzon Y, Trybus KM, Cohen C (1998) Crystal structure of a vertebrate smooth muscle myosin motor domain and its complex with the essential light chain: Visualization of the pre-power stroke state. Cell 94(5):559571

Duke T (2000) Cooperativity of myosin molecules through strain-dependent chemistry. Philos $\mathrm{T}$ Roy Soc B 355(1396):529-538

Edman KAP, Hwang JC (1977) The force-velocity relationship in vertebrate muscle fibres at varied tonicity of the extracellular medium. J Physiol

Eisenberg E, Hill TL (1978) A cross-bridge model of muscle contraction. Progress In Biophysics \& Molecular Biology 33(1):55-82

Erdmann T, Schwarz U (2012) Stochastic force generation by small ensembles of myosin II motors. Phys Rev Lett $108(18)$

Ford LE, Huxley AF, Simmons RM (1977) Tension responses to sudden length change in stimulated frog muscle fibres near slack length. J Physiol 269(2):441-515

Ford LE, Huxley AF, Simmons RM (1981) The relation between stiffness and filament overlap in stimulated frog muscle fibres. J Physiol 311(1):219-249

Fusi L, Brunello E, Reconditi M, Piazzesi G, Lombardi V (2014) The non-linear elasticity of the muscle sarcomere and the compliance of myosin motors. J Physiol 592(5):1109-1118

Givli S (2010) Towards multi-scale modeling of muscle fibers with sarcomere non-uniformities. J Theor Biol 264(3):882-892

Guérin T, Prost J, Joanny JF (2011) Dynamical behavior of molecular motor assemblies in the rigid and crossbridge models. The European Physical Journal E 34(6)

Guo B, Guilford WH (2006) Mechanics of actomyosin bonds in different nucleotide states are tuned to muscle contraction. PNAS 103(26):9844-9849

Hill TL (1974) Theoretical formalism for the sliding filament model of contraction of striated muscle Part I. Prog Biophys Molec Biol 28:267-340

Hill TL (1976) Theoretical formalism for the sliding filament model of contraction of striated muscle part II. Prog Biophys Molec Biol 29:105-159

Hill TL (2004) Free Energy Transduction And Biochemical Cycle Kinetics. Dover

Hill TL, Chen YD (1974) Further analysis of simple prototypal muscle model near and far from equilibrium. PNAS 71(3):2478-2481

Hill TL, Eisenberg E, Chen YD, Podolsky RJ (1975) Some self-consistent two-state sliding filament models of muscle contraction. Biophys J 15(5):335-372

Howard J (2001) Mechanics of Motor Proteins and the Cytoskeleton. Sinauer Associates Incorporated

Hunter PJ, McCulloch AD, ter Keurs HEDJ (1998) Modelling the mechanical properties of cardiac muscle. Progr Biophys Mol Biol 69:289-331

Huxley AF (1957) Muscle structure and theories of contraction. Prog Biophys Mol Bio 7:258-318
Huxley AF, Simmons RM (1971) Proposed mechanism of force generation in striated muscle. Nature 233(5321):533-538

Le Bris C, Lelièvre T (2009) Multiscale modelling of complex fluids: a mathematical initiation. In: Engquist B, Lötstedt P, Runborg O (eds) Multiscale Modeling and Simulation in Science, Springer, Berlin, Heidelberg, pp 49-137

Lecarpentier Y, Chemla D, Blanc FX, Pourny JC, Joseph T, Riou B, Coirault C (1998) Mechanics, energetics, and crossbridge kinetics of rabbit diaphragm during congestive heart failure. The FASEB Journal 12(11):981-989

Linari M, Dobbie I, Reconditi M, Koubassova N, Irving M, Piazzesi G, Lombardi V (1998) The stiffness of skeletal muscle in isometric contraction and rigor: The fraction of myosin heads bound to actin. Biophys J 74(5):2459-2473

Linari M, Caremani M, Piperio C, Brandt P, Lombardi V (2007) Stiffness and fraction of myosin motors responsible for active force in permeabilized muscle fibers from rabbit psoas. Biophys J 92(7):2476-2490

Linari M, Piazzesi G, Lombardi V (2009) The effect of myofilament compliance on kinetics of force generation by myosin motors in muscle. Biophys J 96(2):583-592

Linari M, Brunello E, Reconditi M, Fusi L, Caremani M, Narayanan T, Piazzesi G, Lombardi V, Irving M (2015) Force generation by skeletal muscle is controlled by mechanosensing in myosin filaments. Nature 528(7581):276-279

Lombardi V, Piazzesi G, Linari M (1992) Rapid regeneration of the actin myosin power stroke in contracting muscle. Nature 355(6361):638-641

Lymn RW, Taylor EW (1971) Mechanism of adenosine triphosphate hydrolysis by actomyosin. Biochemistry 10(25):4617-4624

Marcucci L, Truskinovsky L (2010a) Mechanics of the power stroke in myosin II. Phys Rev E 81(5)

Marcucci L, Truskinovsky L (2010b) Muscle contraction: A mechanical perspective. Eur Phys J E 32(4):411-418

Marcucci L, Washio T, Yanagida T (2016) Including thermal fluctuations in actomyosin stable states increases the predicted force per motor and macroscopic efficiency in muscle modelling. PLoS Computational Biology 12(9)

McMahon TA (1984) Muscles, Reflexes, and Locomotion. Princeton University Press

Mobley BA, Eisenberg BR (1975) Sizes of components in frog skeletal muscle measured by methods of stereology. J Gen Physiol 66(1):31-45

Moss RL, Fitzsimons DP (2002) Frank-Starling relationship - long on importance, short on mechanism. Circ Res 90(1):11-13

Niederer SA, Smith NP (2009) The role of the Frank-Starling Law in the transduction of cellular work to whole organ pump function: a computational modeling analysis. PLoS Comput Biol

Offer G, Ranatunga KW (2016) Reinterpretation of the tension response of muscle to stretches and releases. Biophys J 111(9):2000-2010

Peskin CS (1975) Mathematical Aspects of Heart Physiology. Courant Institute of Mathematical Sciences - NYU

Piazzesi G, Francini F, Linari M, Lombardi V (1992) Tension transients during steady lengthening of tetanized muscle fibres of the frog. J Physiol 445:659-711

Piazzesi G, Linari M, Reconditi M, Vanzi F, Lombardi V (1997) Cross-bridge detachment and attachment following a step stretch imposed on active single frog muscle fibres. J Physiol 498:3-15 
Piazzesi G, Lucii L, Lombardi V (2002) The size and the speed of the working stroke of muscle myosin and its dependence on the force. J Physiol 545(1):145-151

Piazzesi G, Reconditi M, Linari M, Lucii L, Bianco P, Brunello E, Decostre V, Stewart A, Gore DB, Irving TC, Irving M, Lombardi V (2007) Skeletal muscle performance determined by modulation of number of myosin motors rather than motor force or stroke size. Cell 131(4):784795

Rayment I, Holden HM, Whittaker M, Yohn CB, Lorenz M, Holmes KC, Milligan RA (1993) Structure of the actinmyosin complex and its implications for muscle contraction. Science 261(5117):58-65

Reconditi M (2006) Recent improvements in small angle xray diffraction for the study of muscle physiology. Rep Prog Phys 69(23):2709-2759

Sainte-Marie J, Chapelle D, Cimrman R, Sorine M (2006) Modeling and estimation of the cardiac electromechanical activity. Computers and Structures 84(28):1743-1759

Sequeira V, Velden J (2017) The Frank-Starling Law: a jigsaw of titin proportions. Biophys Rev pp 1-9

Sheshka R, Truskinovsky L (2014) Power-stroke-driven actomyosin contractility. Phys Rev E 89(1)

Smith DA, Mijailovich SM (2008) Towards a unified theory of muscle contraction. 2: Predictions with the mean-field approximation. Ann Biomed Eng 36:1353-1371

Smith DA, Geeves MA, Sleep J, Mijailovich SM (2008) Towards a unified theory of muscle contraction. 1: Foundations. Ann Biomed Eng 36:1624-1640

de Tombe PP, Mateja RD, Tachampa K, Mou YA, Farman GP, Irving TC (2010) Myofilament length dependent activation. J Mol Cell Cardiol 48(5):851-858

Tortora GJ, Derrikson B (2009) Principles of Anatomy and Physiology, 12th edn. John Wiley \& Sons, Inc

Veigel C, Molloy JE, Schmitz S, Kendrick-Jones J (2003) Load-dependent kinetics of force production by smooth muscle myosin measured with optical tweezers. Nature Cell Biology 5(11):980-986

Washio T, Sugiura S, Kanada R, Okada JI, Hisada T (2018) Coupling Langevin dynamics with continuum mechanics: Exposing the role of sarcomere stretch activation mechanisms to cardiac function. Frontiers in Physiology 9

Zahalak GI (1981) A distribution-moment approximation for kinetic theories of muscular contraction. Mathematical Biosciences 55(1-2):89-114

Zahalak GI (2000) The Two-state Cross-bridge Model of Muscle is an Asymptotic Limit of Multi-state Models. J Theor Biol 204(1):67-82

Zahalak GI, Ma SP (1990) Muscle activation and contraction: constitutive relations based directly on cross-bridge kinetics. J Biomech Eng 112(1):52-62 


\section{A Summary of the main symbols used in the paper}

\begin{tabular}{|c|c|c|}
\hline Symbol & Definition & First occurrence \\
\hline$X^{t}$ & location of the head tip & p. 4 \\
\hline$Y^{t}$ & internal dof of the head (power stroke) & p. 4 \\
\hline$s$ & position of the nearest actin binding site & p. 4 \\
\hline$\alpha^{t}$ & attachment state of the head $(0$ or 1$)$ & p. 4 \\
\hline$\dot{x}_{c}$ & relative velocity of actin filament & p. 4 \\
\hline$u_{\alpha}, w_{\alpha}, u_{e}$ & energy of a myosin head in state $\alpha\left(w_{\alpha}(x, y)=u_{\alpha}(y)+u_{e}(x+y)\right)$ & p. 4 \\
\hline$\eta_{x}, \eta_{y}, \eta$ & drag coefficients & p. 4 \\
\hline & diffusion coefficient & $(2)$ \\
\hline$P_{\alpha}(s, t)$ & probability to be attached $(\alpha=1)$ or detached $(\alpha=0)$ & p.5, (5) \\
\hline$p(x, y, \alpha ; s, t)$ & probability function for $(x, y, \alpha)$ (densities in $(x, y)$, discrete in $\alpha)$ & p.5, (8) \\
\hline $\bar{p}(y ; s, t)$ & effective density in the attached state & $(8)$ \\
\hline$k_{ \pm}$ & attachment $\left(k_{+}\right)$and detachment $\left(k_{-}\right)$rates & (4) \\
\hline$f, g$ & overall attachment $(f)$ and detachment $(g)$ functions & $(20),(21)$ \\
\hline$\mu_{\alpha}$ & chemical potentials for each state & $(22),(23)$ \\
\hline$F$ & free energy & $(24)$ \\
\hline$\dot{S}_{\text {prod }}$ & entropy production rate & p. 8 \\
\hline$\tau_{c}$ & average active tension & $(25)$ \\
\hline $\bar{J}_{1}^{0}$ & flux of the detachment reaction & $(26)$ \\
\hline$\mu_{T}$ & chemical potential of ATP & p. 8 \\
\hline & density functions within the thermal equilibrium hypothesis & (34) \\
\hline$f^{t h}, g^{t h}$ & $\begin{array}{l}\text { overall attachment and detachment function within the thermal equilib- } \\
\text { rium hypothesis }\end{array}$ & $(36 a),(36 b)$ \\
\hline$T_{c}$ & active tension under assumptions on thermal equilibrium and on $f+g$ & p. 10 \\
\hline$K_{c}$ & active stiffness under assumptions on thermal equilibrium and on $f+g$ & p. 10 \\
\hline$\dot{e}_{c}$ & extension rate of sarcomere & p. 10 \\
\hline$\tau_{c}^{t h}$ & $\begin{array}{l}\text { average tension generated by the attached cross-bridges within the ther- } \\
\text { mal equilibrium hypothesis }\end{array}$ & $(46)$ \\
\hline$\check{P}_{1}^{t h}$ & $\begin{array}{l}\text { probability to be attached within the thermal equilibrium hypothesis, in } \\
\text { isometric condition }\end{array}$ & $(43)$ \\
\hline$\check{n}_{1}^{t h}$ & $\begin{array}{l}\text { fraction of attached heads within the thermal equilibrium hypothesis, in } \\
\text { isometric condition }\end{array}$ & $(44)$ \\
\hline$\check{\tau}_{c}^{t h}$ & $\begin{array}{l}\text { average tension within the thermal equilibrium hypothesis, in isometric } \\
\text { condition }\end{array}$ & $(45)$ \\
\hline$T_{0}$ & isometric tension & $(48)$ \\
\hline$K_{0}$ & isometric stiffness & $(48)$ \\
\hline$T_{1}$ & tension reached at the end of a length step & $(49)$ \\
\hline$T_{2}$ & tension reached after the fast isometric transient following a length step & $(50)$ \\
\hline
\end{tabular}

